\title{
RNA-binding protein Mub1 and the nuclear RNA exosome act to fine-tune environmental stress response
}

\author{
Adrien Birot ${ }^{1}$, Krzysztof Kus ${ }^{1}$, Emily Priest ${ }^{1}$, Ahmad Al Alwash ${ }^{1}$, Alfredo Castello ${ }^{1,2}$, Shabaz Mohammed ${ }^{1}$, Lidia Vasiljeva ${ }^{1}$ (D, \\ Cornelia Kilchert ${ }^{3}$ (1)
}

\begin{abstract}
The nuclear RNA exosome plays a key role in controlling the levels of multiple protein-coding and non-coding RNAs. Recruitment of the exosome to specific RNA substrates is mediated by RNAbinding co-factors. The transient interaction between co-factors and the exosome as well as the rapid decay of RNA substrates make identification of exosome co-factors challenging. Here, we use comparative poly(A)+ RNA interactome capture in fission yeast expressing three different mutants of the exosome to identify proteins that interact with poly(A)+ RNA in an exosomedependent manner. Our analyses identify multiple RNA-binding proteins whose association with RNA is altered in exosome mutants, including the zinc-finger protein Mub1. Mub1 is required to maintain the levels of a subset of exosome RNA substrates including mRNAs encoding for stress-responsive proteins. Removal of the zinc-finger domain leads to loss of RNA suppression under non-stressed conditions, altered expression of heat shock genes in response to stress, and reduced growth at elevated temperature. These findings highlight the importance of exosomedependent mRNA degradation in buffering gene expression networks to mediate cellular adaptation to stress.
\end{abstract}

DOI 10.26508/Isa.202101111 | Received 3 May 2021 | Revised 12 November 2021 | Accepted 12 November 2021 | Published online 30 November 2021

\section{Introduction}

Regulation of RNA maturation and degradation is crucial to accurate gene expression (Kilchert et al, 2016). The nucleolytic RNA exosome complex is involved in the biogenesis of multiple types of transcripts produced by RNA polymerases I, II, and III (Pol I, II, and III) (Mitchell et al, 1997; Allmang et al, 1999; Isken \& Maquat, 2007; Kiss \& Andrulis, 2010; Gudipati et al, 2012; Schneider et al, 2012a; Chlebowski et al, 2013; Szczepińska et al, 2015). The nuclear RNA exosome functions in RNA processing ( $3^{\prime}$ end trimming) of stable non-coding (nc) RNA species such as small nuclear and nucleolar RNAs (snRNAs and snoRNAs), ribosomal RNAs (rRNAs), and telomerase RNA, as well as in quality control, where it degrades incorrectly processed
mRNAs and ncRNAs (Mitchell et al, 1997; Allmang et al, 1999; Isken \& Maquat, 2007; Kiss \& Andrulis, 2010; Gudipati et al, 2012; Schneider et al, 2012a; Chlebowski et al, 2013; Coy et al, 2013; Szczepińska et al, 2015; Tseng et al, 2018). The exosome also degrades short-lived nc transcripts produced by Pol II such as promoter upstream transcripts (PROMPTs), enhancer RNAs, and products of wide-spread premature transcription termination in higher eukaryotes, and cryptic unstable transcripts (CUTs) in yeast (Wyers et al, 2005; Preker et al, 2008; Vasiljeva et al, 2008a; Neil et al, 2009; Zhou et al, 2015; Tatomer et al, 2019; Liu et al, 2020). Recent studies have also demonstrated that the exosome not only removes unprocessed mRNAs but also promotes proper mRNA processing because exosome mutants show splicing and mRNA 3' end-processing defects (Nag \& Steitz, 2012; Castelnuovo et al, 2014; Lemay et al, 2014; Shah et al, 2014; Kilchert et al, 2015). Finally, the exosome regulates the levels of specific mRNAs in response to environmental changes and is an important player in executing specific gene expression programmes during development (Harigaya et al, 2006; Chen et al, 2011; Gudipati et al, 2012; Boczonadi et al, 2014; Kilchert et al, 2015; Yang et al, 2020). In various human cell culture models, the activity of the exosome complex was shown to prevent cellular differentiation and maintain cells in an undifferentiated state by suppressing the expression of developmental regulators (Mclver et al, 2014, 2016; Lloret-Llinares et al, 2018; Belair et al, 2019). Mutations in the nuclear exosome lead to severe neurodegenerative diseases in humans, such as spinal muscular atrophy and pontocerebellar hypoplasia (Wan et al, 2012; Boczonadi et al, 2014; Yang et al, 2020).

The nuclear RNA exosome is a $3^{\prime}-5^{\prime}$ exonuclease complex that consists of a nine-protein core (EXO-9) and two catalytic subunits, Rrp6 (EXOSC10) and Dis3/Rrp44 (hDIS3). EXO-9 forms a doublelayered barrel-like structure that comprises six ribonuclease (RNase) PH-like proteins (Rrp41, Rrp42, Rrp43, Rrp45, Rrp46, and Mtr3) and three S1/K homology (KH) "cap" proteins (Rrp4, Rrp40, and Csl4) (Makino et al, 2013). The two catalytic subunits occupy opposite ends of EXO-9 to constitute EXO-11 (Tomecki et al, 2010; Januszyk \& Lima, 2014). Rrp6 is located at the top of the S1/KH cap ring where RNA substrates enter the channel formed by the exosome core, whereas Dis3 is found at the bottom of EXO-9 near the channel exit. Both Rrp6 and Dis3 are $3^{\prime}-5^{\prime}$ exonucleases, but the

${ }^{1}$ Department of Biochemistry, University of Oxford, Oxford, UK ${ }^{2}$ MRC-University of Glasgow Centre for Virus Research, Glasgow, UK ${ }^{3}$ Institute of Biochemistry, JustusLiebig University Giessen, Giessen, Germany

Correspondence: cornelia.kilchert@chemie.bio.uni-giessen.de; lidia.vasilieva@bioch.ox.ac.uk 
latter also has endonucleolytic activity (Chlebowski et al, 2013). In yeast, Rrp6 is restricted to the nucleus, whereas Dis3 is found in both the nucleus and the cytoplasm (Shiomi et al, 1998; Tomecki \& Dziembowski, 2010).

The conserved Ski2-like helicase Mtr4 is essential for RNA degradation by the nuclear exosome (Houseley \& Tollervey, 2009). In the fission yeast, Schizosaccharomyces pombe (S. pombe), Mtr4 shares its function with the highly homologous Mtr4-like helicase Mtl1 (Lee et al, 2013). Mtr4/Mtl1 interact with the exosome cap structure and are thought to play a central role in exosome recruitment to substrate RNAs and the facilitation of RNA degradation through substrate unwinding (Kilchert et al, 2016; Weick et al, 2018; Lingaraju et al, 2019; Weick \& Lima, 2020). In addition to the exosome core, Mtr4/Mtl1 co-purify with RBPs involved in substrate recognition. Mtr4 is a part of the TRAMP complex (Trf4/5-Air1/2-Mtr4), which consists of Mtr4, a zinc-finger protein (Air1 or Air2), and a poly(A) polymerase (Trf4 or Trf5) (LaCava et al, 2005; Vanácová et al, 2005; Bühler et al, 2008). In Saccharomyces cerevisiae, the TRAMP complex is recruited to RNA co-transcriptionally by the RNA- and Pol II-binding protein Nrd1 and mediates degradation of CUTS, among other substrates (Thiebaut et al, 2006; Vasiljeva \& Buratowski, 2006; Vasiljeva et al, 2008a, 2008b; Tudek et al, 2014). In contrast, the human and S. pombe TRAMP complexes appear to be more specialised in regulating rRNA processing (Houseley \& Tollervey, 2009).

In fission yeast, Mtl1 interacts with the conserved YTH domaincontaining protein Mmi1 and its associated proteins, the $\mathrm{C} 3 \mathrm{H} 1$ type zinc-finger protein Red1, the proline-rich protein Iss10, and the enhancer of rudimentary homolog Erh1 (Sugiyama \& SugiokaSugiyama, 2011; Lee et al, 2013; Yamashita et al, 2013; Egan et al, 2014; Zhou et al, 2015). Mmi1 is needed for degradation of a subset of mRNAs encoding for proteins involved in meiosis, cell cycle regulation, and RNA metabolism by the exosome complex and also targets specific ncRNAs. Within these transcripts, Mmi1 is cotranscriptionally recruited to UNAAAC sequence motifs, which can be enriched in regions known as "determinants of selective removal" (DSRs), leading to their rapid degradation by the exosome complex (Harigaya et al, 2006; Chen et al, 2011; Yamashita et al, 2012; Kilchert et al, 2015). The interaction of Mmi1 with Red1 and Mtl1 as a part of the MTREC/NURS (Mtl1-Red1 Core/Nuclear RNA Silencing) complex is important for mediating recruitment of the exosome to RNAs (Shichino et al, 2020). Mmi1 also acts in mRNA quality control and promotes the degradation of inefficiently spliced mRNAs and proper transcription termination of selected transcripts (Shah et al, 2014; Kilchert et al, 2015; Vo et al, 2019). In addition to Mmi1, Iss10, Erh1, and Red1, Mtl1 also co-purifies with other factors that have been functionally linked to exosome regulation: the zf-CCCH-type zinc-finger protein Red5, the poly(A)-binding protein Pab2, the RRM (RNA-Recognition-Motif) and PWI (Pro-Trp-Ile signature) domain-containing protein Rmn1, and the cap-binding proteins Cbc1, Cbc2, and Pir2 (Lee et al, 2013; Zhou et al, 2015). Nevertheless, the mechanisms by which these factors regulate substrate recognition and exosome targeting to substrate RNAs remain poorly understood.

In addition to the RNAs recognised by Mmi1, levels of multiple other transcripts increase in nuclear exosome mutants, suggesting that Mmi1-independent mechanisms contribute to their recognition (Lee et al, 2013; Zhou et al, 2015). We therefore used a quantitative proteomics approach to identify RBPs that are involved in mediating exosome targeting to RNAs. We hypothesised that the association of exosome co-factors with RNA should increase upon stabilisation of their substrate RNAs in the exosome mutants. We compared the poly $(A)+$ transcriptomes and poly $(A)+$ RNA-bound proteomes of control cells and three different exosome mutants (mtl1-1, rrp6s, and dis3-54). Our data suggest that the nuclear exosome plays a more prominent role in controlling the fission yeast transcriptome than previously anticipated. In addition, analysis of the impact of mutations in different exosome subunits on the poly $(A)+-$ bound proteome has identified proteins with increased RNA binding that could function as potential regulators of the exosome in fission yeast. We selected 10 RBPs with significantly altered RNA association and demonstrate that deletion of each of these proteins phenocopies at least one of various phenotypes typical of inactivated nuclear exosome, namely, suppression of transposon RNAs, telomeric silencing, and nuclear RNA retention. We focus on the uncharacterised zf-MYND (MYeloid, Nervy, and DEAF-1)-type zinc-finger protein Mub1, which is highly enriched on poly(A)+ RNA in the exosome mutants. Mub1 physically interacts with the exosome and its deletion leads to increased levels of a specific subset of exosome substrates, supporting its role in exosome regulation.

\section{Results}

\section{Poly(A)+ RNA interactome capture (RIC) in exosome mutants}

We had previously used an unbiased quantitative proteomics approach, RIC, to assess how mutation of various exosome components affects association of the entire complex with poly $(A)+$ RNA (Kilchert et al, 2020a). Specifically, we analysed: rrp6 , lacking exonuclease Rrp6; dis3-54, a Dis3 mutant containing an amino acid substitution (Pro509 to Leu509) within the RNB domain, which reduces its catalytic activity (Ohkura et al, 1988; Murakami et al, 2007); and mtl1-1, a mutant of the helicase Mtl1, which has mutations in the region surrounding the arch domain (Lee et al, 2013) (Fig $1 \mathrm{~A})$. All three mutants are defective in RNA turnover and accumulate known targets of the exosome complex (St-André et al, 2010; Lee et al, 2013; Shah et al, 2014). To gain further insights into the regulation of the exosome complex, we re-analyzed the RIC data to identify RBPs that show increased interactions with poly(A)+ RNA in these mutants. The underlying hypothesis behind this approach was that stabilisation of RNAs targeted by the exosome would facilitate the capture of proteins that are functionally linked to the exosome, which are likely to be enriched on these RNAs (Fig 1B). The three mutant strains were cultured alongside a wild-type (WT) control in the presence of 4-thiouracil $(4 \mathrm{SU})$ to facilitate RNA-protein crosslinking with $365 \mathrm{~nm}$ UV light. After UV crosslinking, poly(A)+ RNA was enriched by oligo-d $(T)$ selection and poly $(A)+$ RNA-associated proteins were identified by mass spectrometry. The abundance of individual proteins in the whole cell extract (WCE) was used to normalise the RIC data as in Garcia-Moreno et al (2019) and Kilchert et al (2020a) (see the Materials and Methods section). The RIC/WCE 


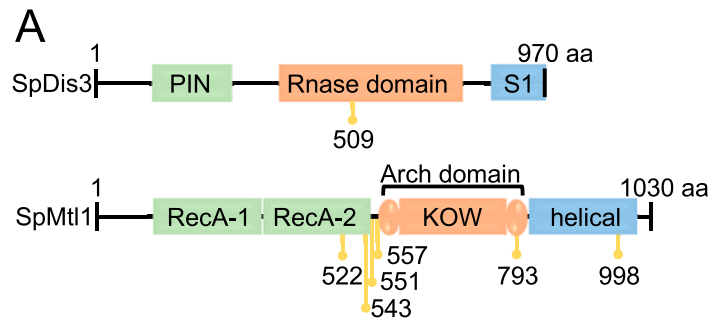

B
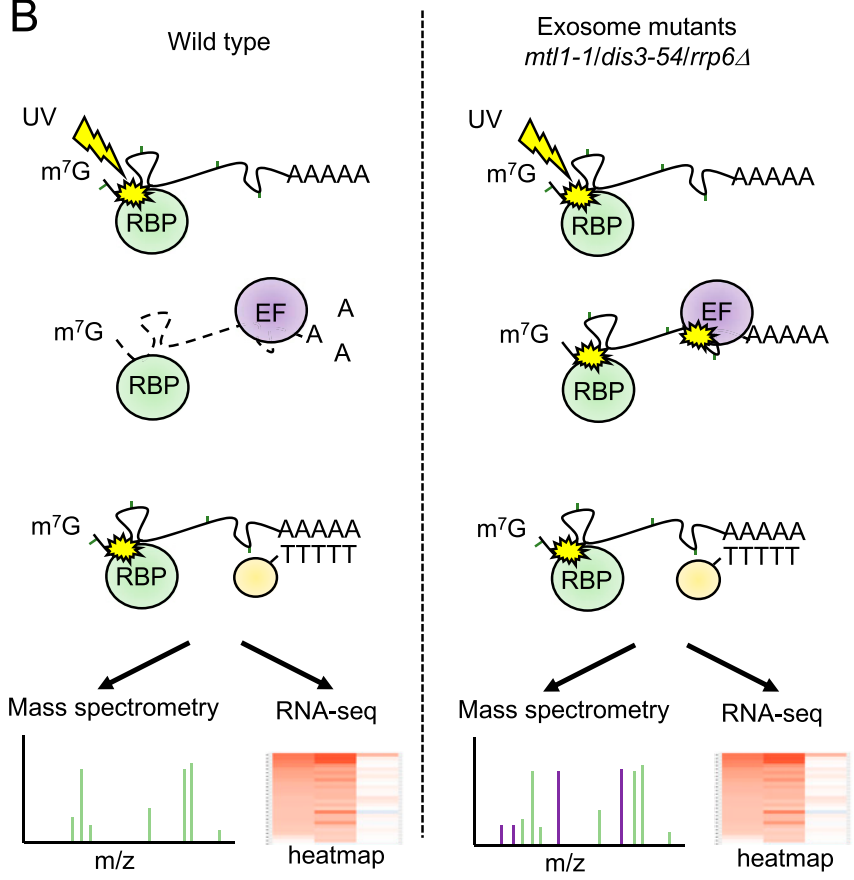

Figure 1. Experimental design of the comparative poly(A)+ RNA interactome capture approach.

(A) Schematic diagram of the domain organisation of S. pombe (Sp) Dis3 and Mtl with the position of the mutations in dis3-54 (P509L) and mtl1-1 (I522M, L543P, Y551H, L557P, D793G, and A998V) indicated in yellow. (B) Schematic diagram of the comparative poly $(A)+$ interactome capture approach. Cells are grown in the presence of 4-thiouracil ( $4 \mathrm{SU})$ and exposed to UV $\left(3 \mathrm{~J} / \mathrm{cm}^{2}\right)$ to allow protein-RNA crosslinking. Poly $(A)+$ RNA and associated proteins are enriched on oligo-d $(T)$ beads and subjected to RNA sequencing and mass spectrometric analysis (RBP, RNA-binding proteins; EF, exosome co-factors involved in recognition of the exosome substrates).

ratio was used to determine the enrichment of each individual protein on poly(A)+ RNA in the mutants relative to the WT (Tables S1 and S2). In addition, RNA sequencing (RNA-seq) was carried out for the oligo-d(T) enriched samples to assess levels of individual poly(A)+ RNAs in WT cells and the exosome mutants.

\section{Exosome RNA targets are enriched in the poly(A)+ RIC samples of the exosome mutants}

First, to confirm that exosome target RNAs were indeed overrepresented in the RIC samples of the different exosome mutants, we analysed their behaviour by RNA-seq. Consistent with the function of the exosome in degradation of ncRNAs (snRNAs, snoRNAs, and CUTs), levels of multiple nc transcripts were increased in all three exosome mutants (Fig $2 \mathrm{~A}$ and Table S3). Compared to rrp6 $\Delta$ and mtl1-1, fewer RNAs in total increased in the dis3-54 mutant (1,373, 1718, and 623 for mtl1-1, rrp6 $\Delta$, and dis3-54, respectively; >1.5-fold, $P$-value $<0.05)$, possibly reflecting that Dis3 function is only partially inhibited under the condition tested. Approximately half of the transcripts with increased levels in the dis3 mutant (54\%) were also dependent on Mtl1, suggesting that these correspond to nuclear targets of the exosome. In agreement with previous reports, transcripts with increased levels in the mutants included many mRNAs (835, 1,078, and 317 for mtl1-1, rrp6 $\Delta$, and dis3-54, respectively; $>1.5$-fold, P-value < 0.05), confirming that the nuclear exosome regulates mRNA levels in addition to its well-described role in degradation of ncRNAs (Fig 2B-D) (Lee et al, 2013; Zhou et al, 2015; Atkinson et al, 2018). However, not all changes necessarily reflect increased mRNA half-lives that are directly related to impaired exosome activity but may be indirect consequences of altered exosome function. The majority of the transcripts that increased in rrp6 $\Delta$ were also increased in $m t / 1-1(\sim 80 \%)$, in agreement with the suggested functional connection between Mtl1 and Rrp6 (Lee et al, 2013; Shichino et al, 2020). Our data support a model where Mtl1 is functionally linked to both exosome-associated nucleases (Rrp6 and Dis3) - with Rrp6 being more dependent on Mtl1 than Dis3 - and confirmed that exosome target RNAs were enriched in the mutant RIC samples.

\section{Mutations in the exosome alter the RNA-bound proteome}

To assess how inactivation of the different subunits of the exosome impacts the RNA-protein interaction profile, we next performed comparative analysis of RBPs differentially enriched in the interactomes of $m t 11-1, \operatorname{rrp} 6 \Delta$ and dis3-54 compared to WT. For each comparative interactome, proteins that were detected in at least two of three biological replicates were included in the analysis (see the Materials and Methods section), resulting in a quantitative data set for a total of 1,146 RBPs. 152, 180, and 83 RBPs were $\geq 2$-fold enriched in $m t 11-1, r r p 6 \Delta$, and dis3-54 over WT, respectively ( $P$-value $<0.1$,Table S2). Consistent with the RNA-seq data, the overlap between RBPs with increased RNA association was substantially larger for rrp6 $\Delta$ and $m t l 1-1$ than for dis3-54 and $m t$ l1-1, further supporting a functional link between Mtl1 and Rrp6 (Fig 2E). Conversely, poly(A)+ RNA association of 137, 95, and 86 RBPs decreased in $m t 11-1$, rrp6 $\Delta$, and dis3-54, respectively ( $P$-value $<0.1$, Fig $2 \mathrm{~F}$ and Table S2). To assess whether RBPs that are differentially regulated in the three exosome mutants are linked to a specific biological process, we performed gene ontology (GO) analysis. The analysis revealed that nuclear RBPs are most noticeably affected in all three exosomes mutants, including dis3-54 where both the nuclear and the cytoplasmic form of the exosome are compromised (Fig $2 \mathrm{G}$ and Table S4). This agrees with the prominent role of the exosome complex in nuclear RNA metabolism. In addition, all mutants exhibit alterations of RBPs involved in "mRNA metabolic process" (GO:0016071) and "ribosome biogenesis" (GO:0042254) (Table S4), suggesting a profound reorganisation of ribonucleoprotein complexes (RNPS) in the exosome mutants. In contrast, the GO term "cytoplasmic translation" (GO:0002181) was significantly overrepresented among the RBPs that contributed less to total RNA-protein interactions in the catalytic exosome mutants than in WT. Indeed, 22 of 95 RBPS that are underrepresented in $\operatorname{rrp} 6 \Delta\left(P\right.$-value $\left.=2.02 \times 10^{-7}\right)$ and 31 


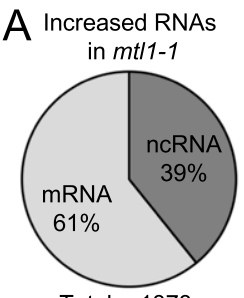

Total $=1373$

Increased mRNAs
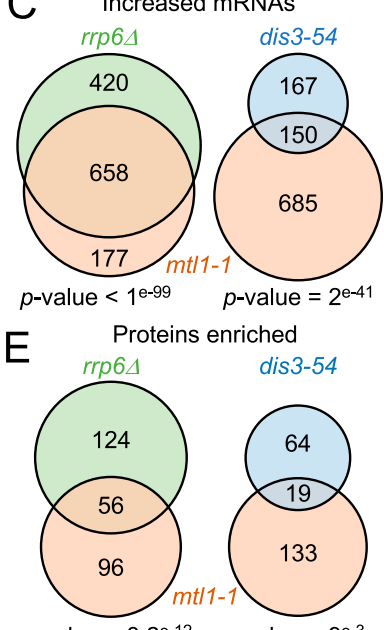

$p$-value $=3.2^{\mathrm{e}-12} \quad p$-value $=9^{\mathrm{e}-3}$

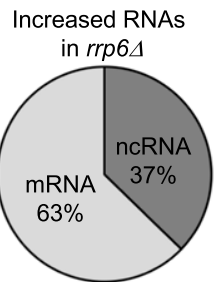

Total $=1718$

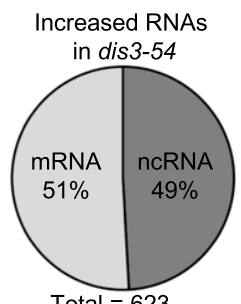

Total $=623$

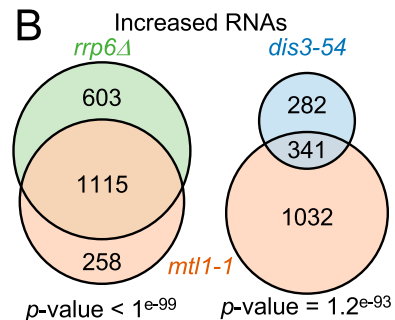

$\mathrm{G}$
D Increased ncRNAs

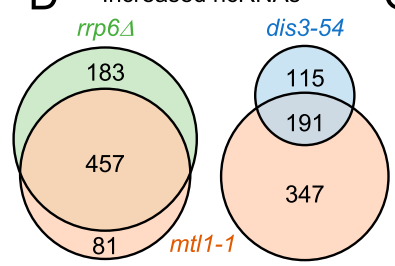

$p$-value $=3.2^{\mathrm{e}-184} \quad p$-value $6.9^{\mathrm{e}-39}$
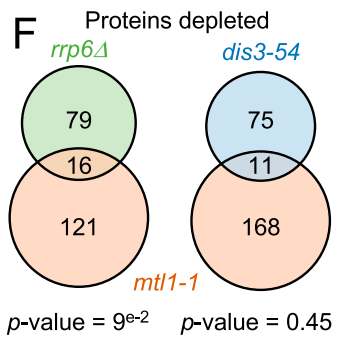

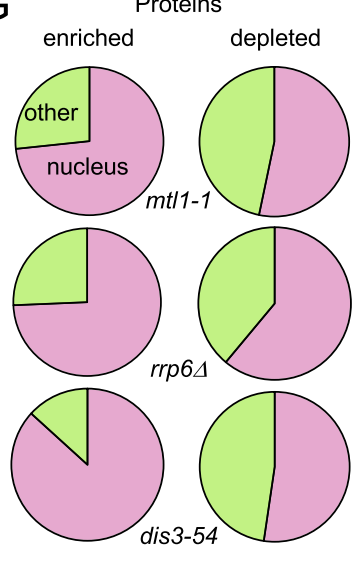

GO cellular component distribution
Figure 2. Effect of exosome mutations on the poly(A)+ RNA transcriptome and protein interactome.

(A) mRNAs and ncRNAs up-regulated (>1.5-fold, P-value $<0.05)$ in $m t l 1-1, r r p 6 \Delta$, and dis3-54 mutants compared to WT. (B) Venn diagram showing overlap between RNAs with increased levels ( $>1.5$-fold, $P$-value $<0.05)$ in $m t l 1-1, \operatorname{rrp} 6 \Delta$, and dis3-54. The P-values indicate the probabilities that the observed overlaps occurred by chance (6,949 genes analysed). (C) Venn diagram showing overlap between mRNAs with increased levels in mtl1-1, rrp6 $\Delta$, and dis3-54 mutants compared with WT (>1.5-fold, $P$-value < 0.05). The $P$-values indicate the probabilities that the observed overlap occurred by chance (5,153 mRNAs analysed). (D) Venn diagram showing overlap between ncRNAs with increased levels in mtl1-1, rrp6 , and dis3-54 mutants compared to WT $(>1.5$-fold, $P$-value $<0.05)$. The $P$-values indicate the probabilities that the observed overlap occurred by chance (1,796 ncRNAs analysed). (E) Venn diagram showing proteins that are enriched in poly $(A)+$ RNA pull-downs of $m$ tl1-1 and either rrp6 $\Delta$ or dis3-54 relative to $W T$. The $P$-values indicate the probabilities that the observed overlap occurred by chance $(1,146$ proteins analysed). (F) Venn diagram showing proteins depleted from the poly(A)+ pull-down in mtl1-1 and either rrp6 $\Delta$ or dis3-54 relative to WT. The P-values indicate the probabilities that the observed overlap occurred by chance (1,146 proteins analysed). (G) RNA association of nuclear proteins (GO:0005575) is strongly affected in the exosome mutants. of 86 RBPs that are underrepresented in dis3-54 ( $P$-value = $\left.7.16 \times 10^{-17}\right)$ are annotated with "cytoplasmic translation." Altogether, RIC analyses of exosome mutants demonstrate significant changes in protein-RNA interactions in exosome-deficient cells, highlighting the important role of the exosome in nuclear RNP metabolism.

\section{Exosome mutants show different patterns of poly(A)+ RNA accumulation in the nucleus}

The strong increase in the association of poly(A)+ RNA with RBPs in the nucleus combined with the lower association with proteins involved in cytoplasmic translation may reflect a change in subcellular localisation of poly $(A)+$ RNA in these strains. Indeed, it was previously reported that poly $(A)+$ RNA is retained in the nucleus in rrp6 mutants (Paul \& Montpetit, 2016; Fan et al, 2017; Silla et al, 2018).

To assess whether all three exosome mutants exhibit increased levels of poly $(A)+$ RNA in the nucleus, we performed FISH with oligo(dT) probes. Poly(A)+ RNA accumulated inside the nucleus in all three mutants (Fig 3A). Quantification of the FISH signal confirmed a phenotype of nuclear RNA retention manifested by a higher nuclear/cytoplasmic ratio compared to WT, with the strongest effect observed for rrp6 (Fig 3B and Table S5). However, careful observation revealed different patterns of poly $(A)+$ RNA accumulation in the three mutants (Fig 3A): In dis3-54, a diffuse nuclear signal is observed. In contrast, distinct poly $(A)+$ RNA foci are detected inside the nuclei of $m$ tl1-1 cells. Last, $\operatorname{rrp} 6 \Delta$ cells show a mixed phenotype with foci present in addition to a strong diffuse nuclear poly(A)+ FISH signal. We had previously reported that RNA association with the exosome complex is increased in dis3-54 (reflecting its inability to efficiently degrade RNA substrates), whereas RNA association with the exosome complex is strongly reduced in mtl11, suggesting a disruption of substrate recruitment as well as decay (Kilchert et al, 2020a). This blockage at different stages of the RNA degradation process may be responsible for the different behaviour of the retained transcripts. An accumulation of exosome target RNAs in nuclear foci has been observed upon inactivation of hMTR4 or its co-factors in human cells (Meola et al, 2016; Fan et al, 2018).

\section{Comparative RIC identifies known exosome co-factors}

To validate our hypothesis that exosome co-factors are indeed differentially enriched in the comparative interactome capture experiment, we first analysed how RNA association of Mmi1 is affected in the exosome mutants. As expected, the association of Mmi1 with poly $(A)+$ RNA increased two and fourfold in mtl1-1 and $\operatorname{rrp} 6 \triangle \mathrm{RIC}$, respectively, compared with WT cells ( $P$-value = 0.039 and $P$-value $=0.001)$; in dis3-54, the change was not significant $(>1.5$-fold, $P$-value $=0.768)($ Fig $3 C)$. The increased RNA association of Mmi1 correlated with an increased abundance of Mmi1 RNA substrates in the exosome mutants. Wellcharacterised meiotic RNA targets of Mmi1 were most strongly enriched in $\operatorname{rrp} 6 \Delta$ cells, significantly enriched in the mtl1-1 mutant, and only mildly affected in dis3-54 (Fig 3D). Moreover, the relative poly $(A)+$ RNA association of other known exosome co-factors also increased in the exosome mutants. For example, 
A

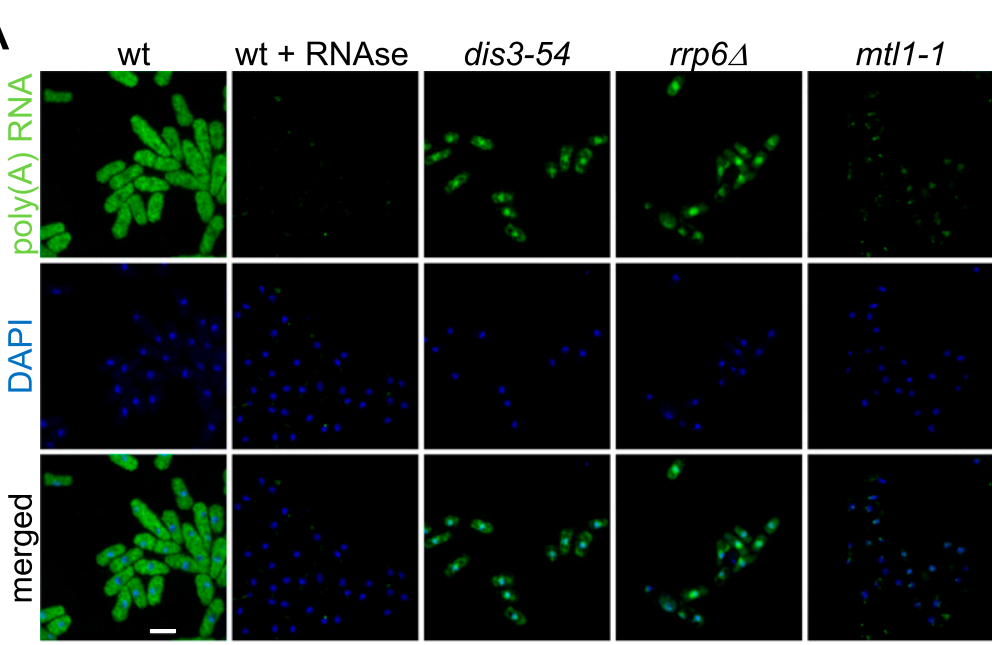

B Poly $(A)+F I S H$ signal quantification
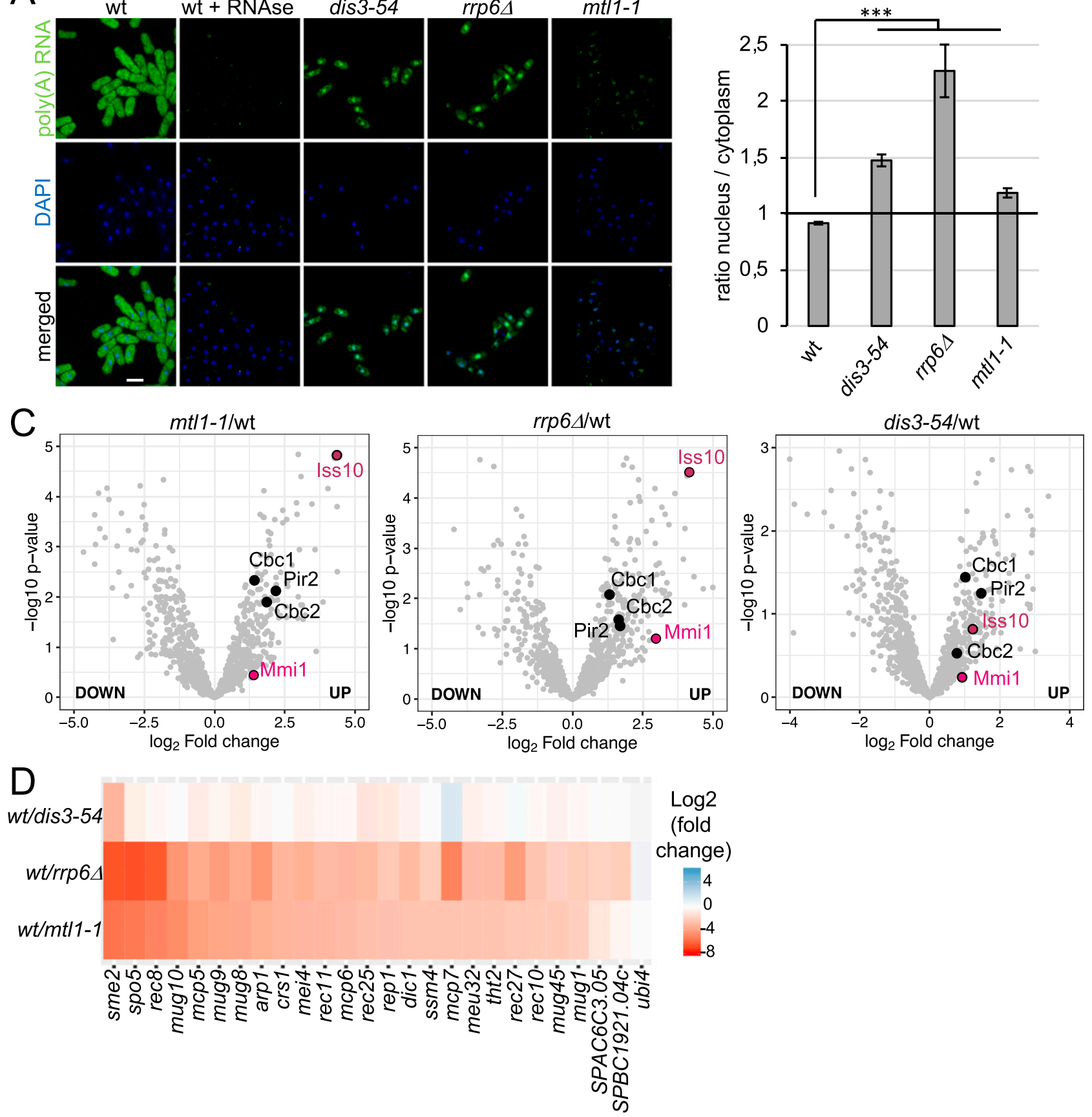

Figure 3. Poly(A)+ RNA accumulates in the nucleus of exosome mutants and RBPs with functional links to the exosome are enriched on poly(A)+ RNA.

(A) Poly(A)+ RNA FISH analysis of rrp6 , dis3-54, and $m$ tl1-1 cells. Nuclear co-staining with DAPI is shown in blue. Poly(A)+ RNA is visualised in green. Scale bar $=10 \mu m$. (B) Quantification of the poly(A)+ RNA FISH experiments shown in Fig 3A ( $n=30-60$ cells, *** $P$-value $<0.0001$, un-paired $t$ test). (C) Volcano plots showing enrichment of Mmi1, Iss10, Cbc1, Cbc2, and Pir2 (S. pombe ARS2) on poly(A)+ RNA in the three exosome mutants. In the volcano plot, $P$-values (-log10, moderated $t$ test) are plotted against the ratio of $\log _{2}$-fold changes in mass spectrometry (MS) intensities for the whole-cell extract-normalised proteomes of mutant versus WT cells recovered from the oligo-d(T) pull-downs of UV-crosslinked samples $\left(3 \mathrm{~J} / \mathrm{cm}^{2}\right)$. In all panels, individual proteins are depicted as a single dot. (D) Heat map analysis of poly(A)+ RNA-seq showing differential expression of Mmi1 regulon RNAs in exosome mutants compared with WT.

RNA association of Iss10, an MTREC component, increased between 2-fold and more than 10-fold in dis3-54, mtl1-1, and rrp6 over WT $\left(P\right.$-value $=0.15, P$-value $=3.26 \times 10^{-5}$ and $P$-value $\left.=1.73 \times 10^{-5}\right)$. Similarly, RNA association of the nuclear cap-binding complex $\mathrm{Cbc1}$,
Cbc2 and Pir2 increased (>4-fold) in all three mutants (Table S1) Together, this illustrates the capacity of comparative RIC to discover RBPs with bona fide roles in exosome targeting to substrate RNAs. 


\section{Identification of RBPs with functional links to the exosome}

To uncover cellular RBPs with novel roles in exosome regulation, we selected 10 proteins without well-described functions in RNA metabolism that have a classical RNA-binding domain (Lunde et al, 2007) and whose RNA association was increased ( $>2$-fold) in the exosome mutants (Figs 4A and S1A). We generated genomic deletions of the candidate RBPS to test whether these recapitulate phenotypes associated with compromised exosome function. First, we assessed whether nuclear retention of poly(A)+ RNA is observed in any of the deletion strains using RNA FISH (Figs 4B and S1B). In 8 of 10 deletion strains, the nuclear/cytoplasmic ratio of the poly $(A)+$ RNA FISH signal was significantly increased relative to WT (Fig 4C and Table S6), with the strongest effect observed for SPCC126.11c $\triangle$ (ratio of poly $(A)+$ signal $~ 1.5$ ). In all cases, the pattern of RNA accumulation in the nucleus resembled the diffuse staining of rrp6 $\Delta$ and dis3-54 rather than the pronounced punctate staining characteristic of the mtl1-1 mutant (compare Fig 3A). No nuclear RNA retention was observed upon deletion of mub1 or SPABC18H10.09.

The exosome complex is also required for robust heterochromatic gene silencing in S. pombe, resulting in the accumulation of heterogeneous RNAs produced from telomeric and centromeric regions of the genome in exosome mutants (Bühler et al, 2007; Lee et al, 2013). To test whether the candidate RBPs contribute to the repression of heterochromatic transcripts, the deletion mutants were crossed into a strain background with a ura4+ reporter inserted within the transcriptionally silent telomeric region of chromosome I. We then monitored the ability of the reporter strains to grow on -URA plates and plates containing 5-fluoroorotic acid (5FOA), a compound that is toxic when a functional version of the ura 4 gene is expressed. The deletion of either SPCC126.11C, srp40, or SPAC222.18 led to moderate growth on -URA plates and attenuated growth on 5-FOA compared with WT (Figs 4D and S1C), suggesting that these RBPs may function in heterochromatin formation or maintenance.

To assess whether any of the 10 candidate RBPs contributes to regulating levels of exosome substrate RNAs, we determined the steady-state levels of the RNA produced from the Tf2 retrotransposable element (SPAC9.04). Tf2-1 is up-regulated in rrp6 $\triangle$ but independent of Mmi1 (Fig 4E, lanes 1-5). Only in mub1 $\triangle$ cells was a pronounced increase in tf2-1 RNA levels observed, suggesting that, similar to Rrp6, Mub1 may contribute to the suppression of RNAs derived from retrotransposable elements (Fig 4E).

\section{Mub1 controls levels of stress-induced mRNAs targeted by the exosome}

Because deletion of mub1 led to increased levels of known exosome targets such as tf2-1 RNA, we investigated the involvement of the protein in RNA metabolism in more detail. Fission yeast Mub1 is the homolog of $\mathrm{S}$. cerevisiae multi-budding 1 and contains an Armadillo-type domain and a potential nucleic acid-binding region (zf-MYND domain). To assess the contribution of Mub1 to regulation of RNA levels, we compared the transcriptomes of WT and mub1A cells by RNA-seq normalised to S. cerevisiae spike-in (see the Materials and Methods section). The levels of 248 transcripts ( 162 mRNAs and 86 ncRNAs) increased more than 1.5-fold ( $P$-value $<0.05$ ) in mub1 $\triangle$ cells (Fig 5A and Tables S7 and S8). GO term analysis revealed a significant enrichment of "Core Environmental Stress Response induced (CESR)" genes (46.25\% (74/160), P-value $=1.55246 \times 10^{-28}$, AnGeLi tool [Bitton et al, 2015]). 144 transcripts (92 mRNAs and 52 ncRNAs) affected by Mub1 deletion were also up-regulated in mtl1-1 (Figure 5A and B), in support of a possible functional link between Mtl1 and Mub1. The GO term "Core Environmental Stress Response induced" was strongly overrepresented among mRNAs that were upregulated in both mutant strains (64.44\% (58/90), $P$-value $=3.32315 \times 10^{-32}$, AnGeLi tool [Bitton et al, 2015]), potentially indicating a role for Mub1 and Mtl1 in repressing the stress response under non-stress conditions. To validate the RNA-seq data, increased steady-state levels of two representative CESR mRNAs (Chen et al, 2003), gst2 and SPCC663.08c, in mub1D and mtl1-1 mutants were confirmed by Northern blot analyses (Fig 5B). No additive effect was observed in the double mutant mtl1-1 mub1 $1 \Delta$ compared with the single mutants, which is consistent with Mtl1 and Mub1 acting in the same pathway (Fig 5B).

\section{The MYND-type zinc finger domain is required for regulation of RNA levels}

Our observation that Mub1 was enriched in the poly(A)+ RNA interactome suggested that Mub1 is an RBP. We hypothesised that the zf-MYND domain (471-528) could mediate an interaction with RNA. Multiple other proteins that associate with the exosome contain zinc-finger domains, for example Red1, Red5, and Air1, a component of the TRAMP complex (Keller et al, 2010; Sugiyama \& Sugioka-Sugiyama, 2011; Sugiyama et al, 2013). For Red1 and Red5, the zinc-finger domains were shown to be important for RNA degradation by the exosome as mutations introduced in these domains lead to RNA stabilisation (Sugiyama \& Sugioka-Sugiyama, 2011; Sugiyama et al, 2013). To test whether the zf-MYND domain is required for Mub1 function, we deleted the region between amino acids 471 and 528 (Fig 5C). Deletion of the zf-MYND domain led to the expected change in the size of the protein, which we assessed by visualising FLAG-tagged protein by Western blot (Fig 5D). Addition of a triple FLAG tag to full-length Mub1 did not have any effect on cellular protein levels or cell growth, nor did it induce the spheroid cell shape typical of a mub1 deletion (Hayles et al, 2013) (Figs 5E and S2A and B). The protein level of Mub1 $\triangle 471-528-3 \times F L A G$ (Mub1- $\triangle Z$ 3xFLAG) was comparable to Mub1-3xFLAG, suggesting that deletion of the zf-MYND domain did not affect the stability of the truncated protein (Fig 5D). To test the importance of the zinc-finger domain for regulation of mRNA levels, we assessed levels of gst2 and SPCC663.08c mRNAs in the mub1- $\triangle Z-3 x F L A G$ mutant by Northern blot. Similar to the phenotype observed for mub1 $\triangle$, expression of both mRNAs increased in the mub1- $\triangle Z-3 X F L A G$ mutant compared with WT cells (Fig 5F), suggesting that the zf-MYND domain is essential for the function of Mub1 in RNA metabolism. At present, we do not know to what extent disruptions of either protein-RNA or protein-protein interactions contribute to the mutant phenotype. Cells expressing the truncated Mub1- $\Delta Z-3 x$ FLAG display the characteristic rounded shape of mub1 $\triangle$ cells (Fig S2A and data not shown), a phenotype that is not observed for exosome mutants, suggesting that additional, exosome-independent functions of the protein might also be affected in this mutant. 
A

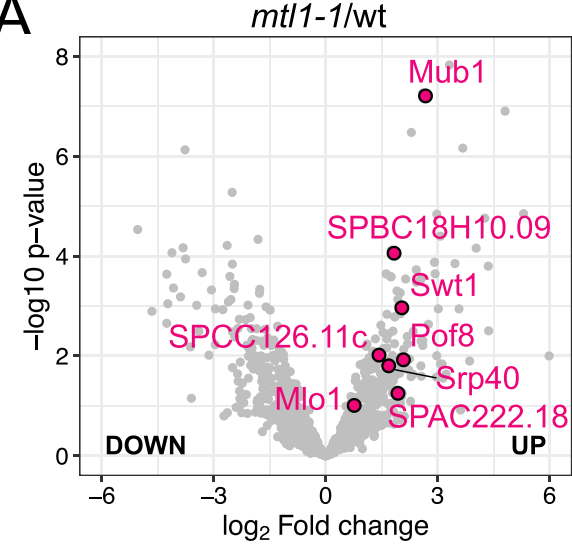

B
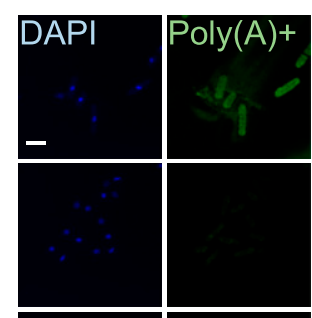

Merged
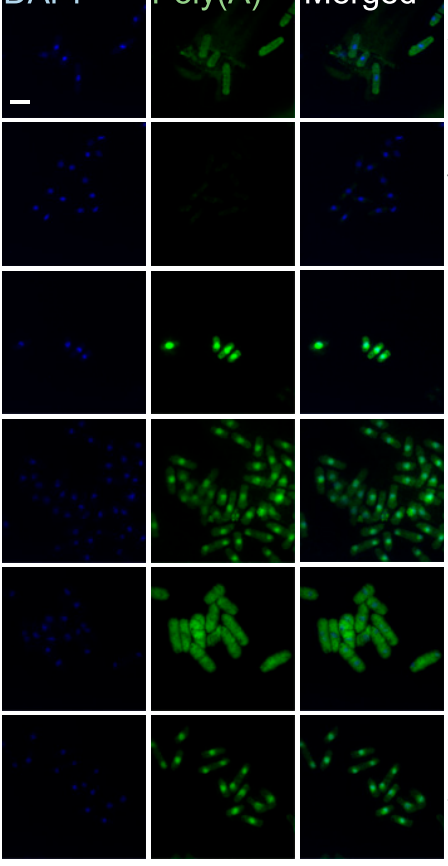

wt

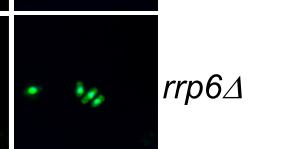

wt +

RNAse A
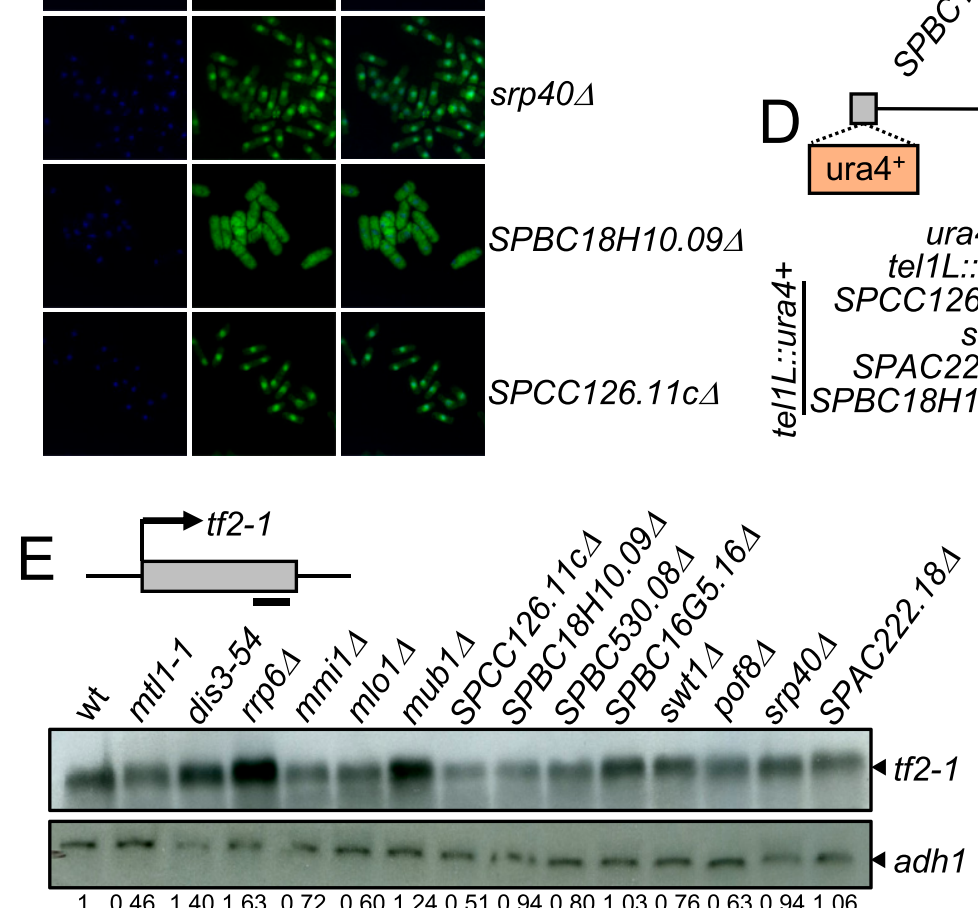

Figure 4. Identification of RBPs with functional links to the exosome.

(A) Volcano plot showing the relative enrichment of the candidate proteins on poly(A)+ RNA in mtl1-1, rrp6 4 , and dis3-54 as in 3C. (B) Poly(A)+ RNA FISH analysis of the indicated strains. Nuclear co-staining with DAPI is shown in blue. Poly(A)+ RNA is visualised in green. Scale bar = $10 \mu \mathrm{m}$. (C) Quantification of the poly(A)+ RNA FISH experiments shown in Figs 4B and S1B ( $n=25-60$ cells, ${ }^{* * *}$-value $<0.0001$, n.S., not significant, un-paired $t$ test). (D) Serial dilutions of the indicated strains spotted on complete medium supplemented with adenine (YES+A), medium lacking uracil (-URA) or containing 5-FOA (5-FOA) and grown at 25 ${ }^{\circ} \mathrm{C}$. A schematic representation of Chromosome I (Chr I) with centromeric and telomeric regions (grey circle and grey rectangles, respectively) is included. The position of the $\mathrm{ura}^{+}{ }^{+}$reporter in the left arm of 


\section{Disruption of Mub1 function leads to sensitivity to heat stress}

We had observed that genes involved in the core environmental stress response-many of which are induced during heat shock-are dysregulated in mub1 $\Delta$ and mtl1-1 already under nonstressed conditions (see above). To test whether the dysregulation of stress-responsive transcripts extended to stress conditions, we performed Northern blot for hsp16 mRNA, which encodes a heat shock protein (Hirose et al, 2005). Under non-stressed conditions, no detectable RNA is produced from the hsp16 locus in WT cells; however, several bands can be detected in mub1 $\Delta$ and $m$ tl1-1 (Fig $S 2 C$ and $D$, lanes 1-4). The lower band corresponds to the mature mRNA in size, whereas the upper bands may represent isoforms with longer 5' or 3'UTRs. As expected, levels of hsp16 mRNA sharply increased in WT cells after induction of the heat shock response $(4 \mathrm{~h}$ at $37^{\circ} \mathrm{C}$ ). However, heat shock-dependent induction of hsp16 mRNA was amplified in mub1 1 , mtl1-1, and the double mutant (Fig S2D, compare lanes 6-8 to lane 5). Please note that the hsp16 isoforms that are stabilised in the mutants under non-stressed conditions are absent at $37^{\circ} \mathrm{C}$, potentially reflecting a higher complexity in hsp16 regulation, which needs to be further characterised. When we assayed the comparative fitness of the different strains under conditions of heat stress, we observed that growth at $37^{\circ} \mathrm{C}$ is impaired in mub1 $\triangle$ and mub1- $\triangle Z-3 x F L A G$ (Fig 5E). We consider it plausible that the dysregulation of expression of stress-responsive genes contributes to the increased sensitivity of mub1 mutant strains to high temperatures, although we cannot exclude that Mub1 might also promote cellular adaptation to suboptimal temperatures through other mechanisms.

\section{Mub1 regulates levels of stress-responsive transcripts at a post-transcriptional level}

We observed a strong de-repression of many CESR genes, including gst2, SPCC663.08c, and hsp16, in mub1 $\triangle$ and mtl1-1 mutants. To test whether mutation of mub1 triggers a transcriptional induction of the CESR under normal growth conditions, we assessed Pol II occupancy at these genes by chromatin immunoprecipitation (Pol II ChIP). We observed no significant change in Pol II occupancy in mub1 $\triangle$ and mub1- $\triangle Z-3 x$ FLAG compared with WT cells (Fig 6A and Table S9). These results strongly suggest that Mub1 contributes to regulation of stress-responsive transcripts at the posttranscriptional level.

To assess whether Mub1 and the exosome complex physically interact, we performed co-immunoprecipitation experiments. Mub1 co-purified with the Rrp6 subunit of the exosome, supporting a physical link between Mub1 and the nuclear exosome (Fig 6B). The reverse co-immunoprecipitation yielded the same result (Fig 6C). Importantly, the interaction between Rrp6 and Mub1 is no longer detected upon RNAse A treatment (Fig 6C). These data support a connection between Mub1 and the exosome complex that is mediated by RNA and is compatible with Mub1 acting as a co-factor of the complex.

\section{Discussion}

Taken together, our comparative RIC analysis identified several proteins that may function as potential exosome regulators, including Mub1, SPCC126.11c, Srp40, or SPAC222.18, whose deletion phenocopies key aspects of exosome dysfunction. Based on the correlated phenotypes, our data suggest a relationship between these factors and the nuclear RNA exosome. However, further work is needed to fully explain and understand how these factors might be functionally connected. Interestingly, SPCC126.11c and Srp40, which we did not further characterize, were previously reported to co-purify with the exosome, suggesting a direct functional link between these proteins and the exosome (Egan et al, 2014; Telekawa et al, 2018). SPCC126.11c has some similarity to ALYREF and CHTOP, components of the human TRanscription and EXport (TREX) complex that also co-purify with hMTR4 in human cell lines (Gaudet et al, 2011; Andersen et al, 2013). Moreover, a recent study reports that SRSF3, the human orthologue of SPAC222.18, is involved in exosome recruitment via the Nuclear Exosome Targeting (NEXT) complex, raising the possibility that the role of the serine-rich protein SPAC222.18 is conserved in S. pombe (Mure et al, 2018). In particular, our data highlight a role for Mub1 and the exosome complex in suppressing the heat shock response, potentially through rapid turnover of stress-responsive mRNAs under nonstressed conditions. A reverse mechanism was recently described in budding yeast, where the Rrp6 component of the exosome was found to be required for the full induction of cell wall integrity (CWI) genes, thereby promoting cell survival during heat shock (Wang et al, 2020). Similarly, the exosome was shown to be recruited to heat shock genes in heat-stressed Drosophila Kc cells (Andrulis et al, 2002). This suggests that a function of the exosome in modulating environmental adaptation is conserved and can act on multiple levels. Further works will assess by which mechanism Mub1 contributes to this regulation.

\section{Materials and Methods}

\section{Yeast strains and manipulations}

General fission yeast protocols and media are described in Moreno et al (1991). All strains are listed in Table S10. Cells were grown in YES medium at $30^{\circ} \mathrm{C}$ unless stated otherwise. Gene deletions and epitope tagging were carried out by homologous recombination (Bähler et al, 1998). All oligos are listed in Table S11. Protein extracts and Western blotting were performed as described in Feytout et al (2011).

the telomere is indicated. (E) Analysis of tf2-1 mRNA levels by Northern blot in the indicated strains. Band corresponding to tf2-1 is indicated with an arrow. Schematic shows location of the probe (black bar). Adh1 mRNA is included as a loading control. Numbers indicate the relative abundance of tf2-1 RNA normalised to adh1 (technical replicate $n=2)$. 
A

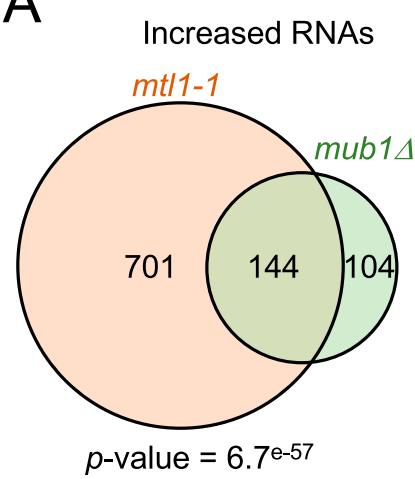

C
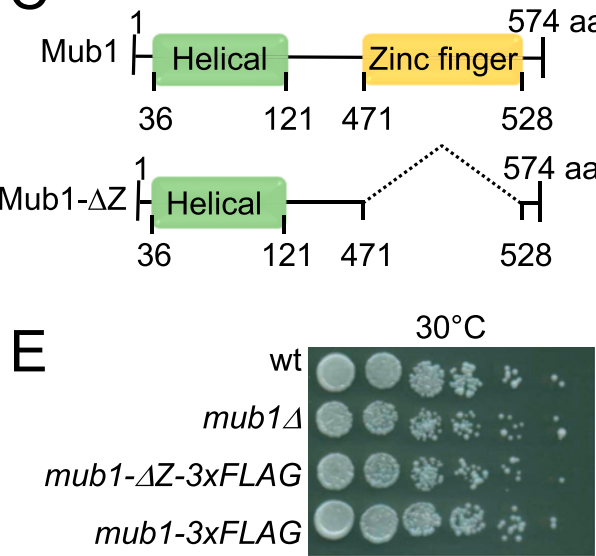
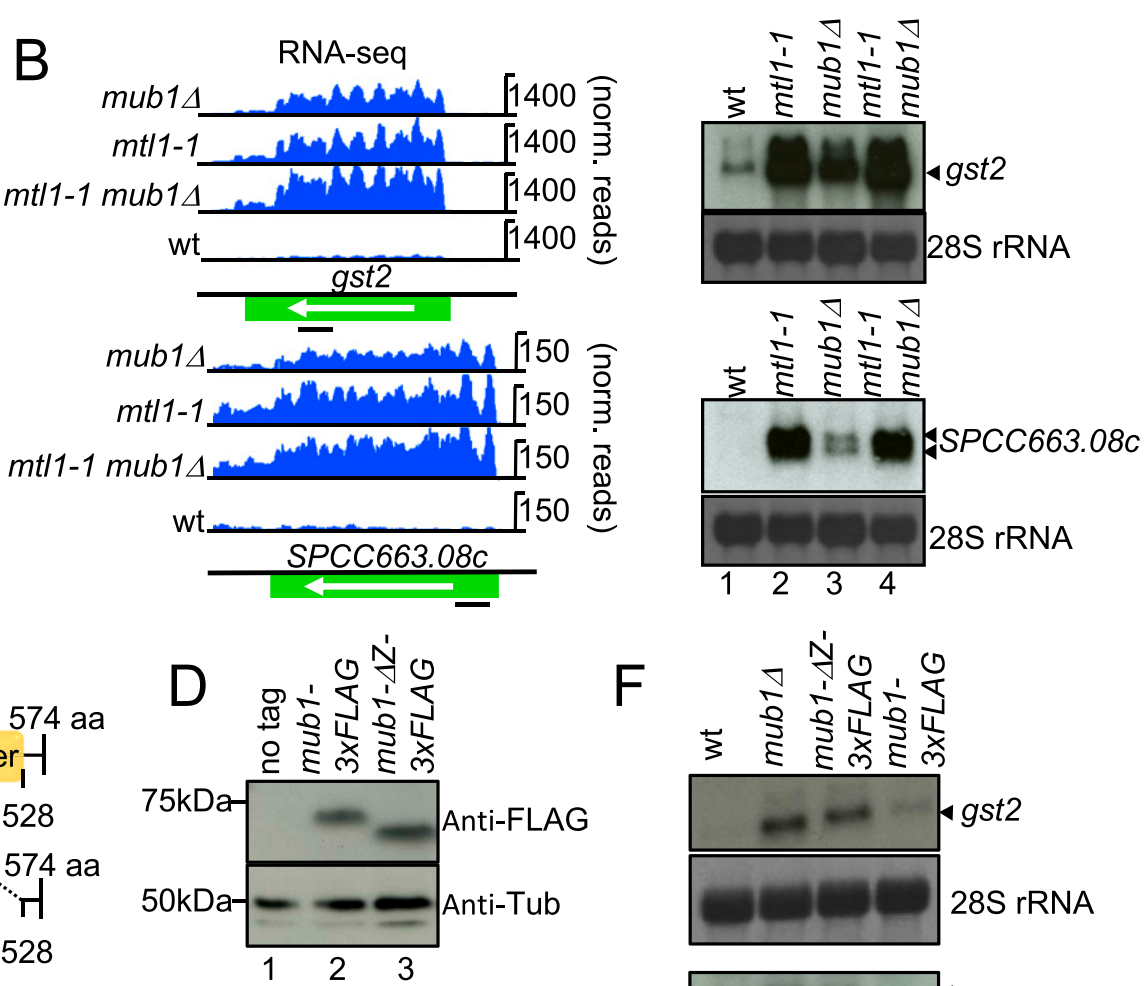

$\mathrm{F}$
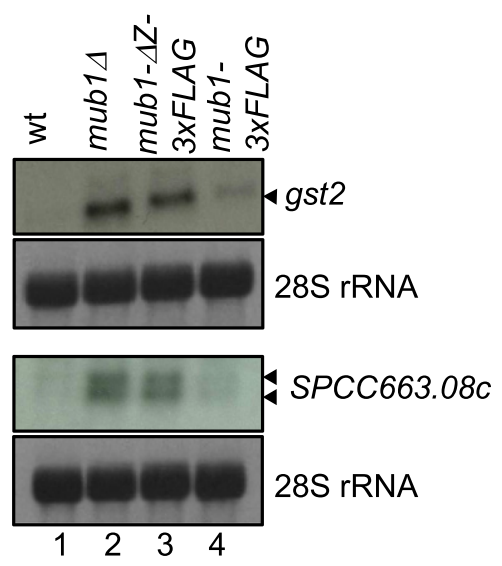

Figure 5. Mub1 protein functions in maintaining levels of exosome RNA targets.

(A) Venn diagram showing overlap between RNAs that increase in levels in mtl1-1 and mub1 $\Delta$ (>1.5-fold, P-value <0.05). (B) Genome browser snapshots showing RNAseq data and Northern blot analysis for two representative transcripts (gst2 and spcc663.08c) up-regulated in mtl1-1 and mub1 $\triangle$ compared with WT. Bands corresponding to gst2 and spcc663.08c are indicated with arrows. Positions of the probes are indicated with black bars. 28S ribosomal RNAs (rRNA) stained with methylene blue are included as loading control (biological replicate $n=3$ ). (C) Graphical representation of Mub1 and Mub1- $\Delta Z$. (D) Western blot against FLAG to assess levels of endogenously expressed Mub1-3xFLAG and Mub1- $\triangle Z$-3xFLAG proteins (biological replicates $n=2$ ). (E) Serial dilutions of the indicated strains spotted on complete medium (YES+A) at the indicated temperatures (biological replicates $n=2$ ). (B, F) Northern blot analysis of SPCC663.08c and gst2 mRNAs as in (B), (biological replicates $n=2)$.

\section{Co-immunoprecipitation}

To prepare WCEs, cells were collected, rinsed in ice-cold PBS with1 $\mathrm{mM}$ PMSF, and frozen on dry ice. Lysis was performed in $125 \mu \mathrm{l}$ icecold lysis buffer (50 mM Tris, pH 7.5, $150 \mathrm{mM} \mathrm{NaCl}, 1 \mathrm{mM} \mathrm{MgCl} 2,0.5 \%$ Triton X-100, 1 mM DTT, and 10\% glycerol) with inhibitors (complete EDTA free protease inhibitor, 40694200; Roche, and 1 mM PMSF) using a bead beater (Cryoprep). After addition of $275 \mu$ lice-cold lysis buffer with protease inhibitors, the extracts were clarified by two successive rounds of centrifugation. Samples were incubated for $1 \mathrm{~h}$ at $4^{\circ} \mathrm{C}$ with anti-MYC magnetic beads (TA150044; Origene). Beads were washed five times with lysis buffer without inhibitors. Proteins were eluted with hot Laemmli buffer at $95^{\circ} \mathrm{C}$ for $5 \mathrm{~min}$. For reverse co-immunoprecipitation, lysis was performed in ice-cold buffer (50 mM Hepes, pH 7.6, 75 mM KCl, 1 mM MgCl 2,1 mM EGTA, 1 mM DTT, 0.1\% Triton X-100, $10 \mathrm{mM}$ Sodium butyrate, and 10\% glycerol) with inhibitors (1 mM PMSF, 1 mM NaVa, 10 mM $\beta$-glycerophosphate, and $1 X$ protease inhibitor [P8215; Sigma-Aldrich]). Samples for each strain were distributed to two tubes. One was treated with $2.5 \mu \mathrm{g}$ of RNase A (R6513; Sigma-Aldrich) for 20 min on ice. Samples were immunoprecipitated for $1 \mathrm{~h}$ at $4^{\circ} \mathrm{C}$ with anti-FLAG antibody (F1804; SigmaAldrich) coupled to $\mu$ MACS protein G (130-071-101; Miltenyi Biotec).

\section{Northern blotting}

Northern blot experiments were essentially performed as described (Vasiljeva \& Buratowski, 2006). RNA was prepared as described in Kilchert et al (2015). $8 \mu \mathrm{g}$ of RNA were resolved on a 1.2\% agarose gel containing $6.7 \%$ formaldehyde in MOPS buffer. After capillary transfer in $10 \times$ SSC onto a Hybond $\mathrm{N}+$ membrane (GE Healthcare), RNA was UV-crosslinked and stained with methylene blue to visualise ribosomal RNAs. Gene-specific probes were generated by random priming in the presence of ATP $\left[\alpha^{32} P\right]$ using the Prime-It II Random Primer Labeling Kit (300385; Agilent) using 

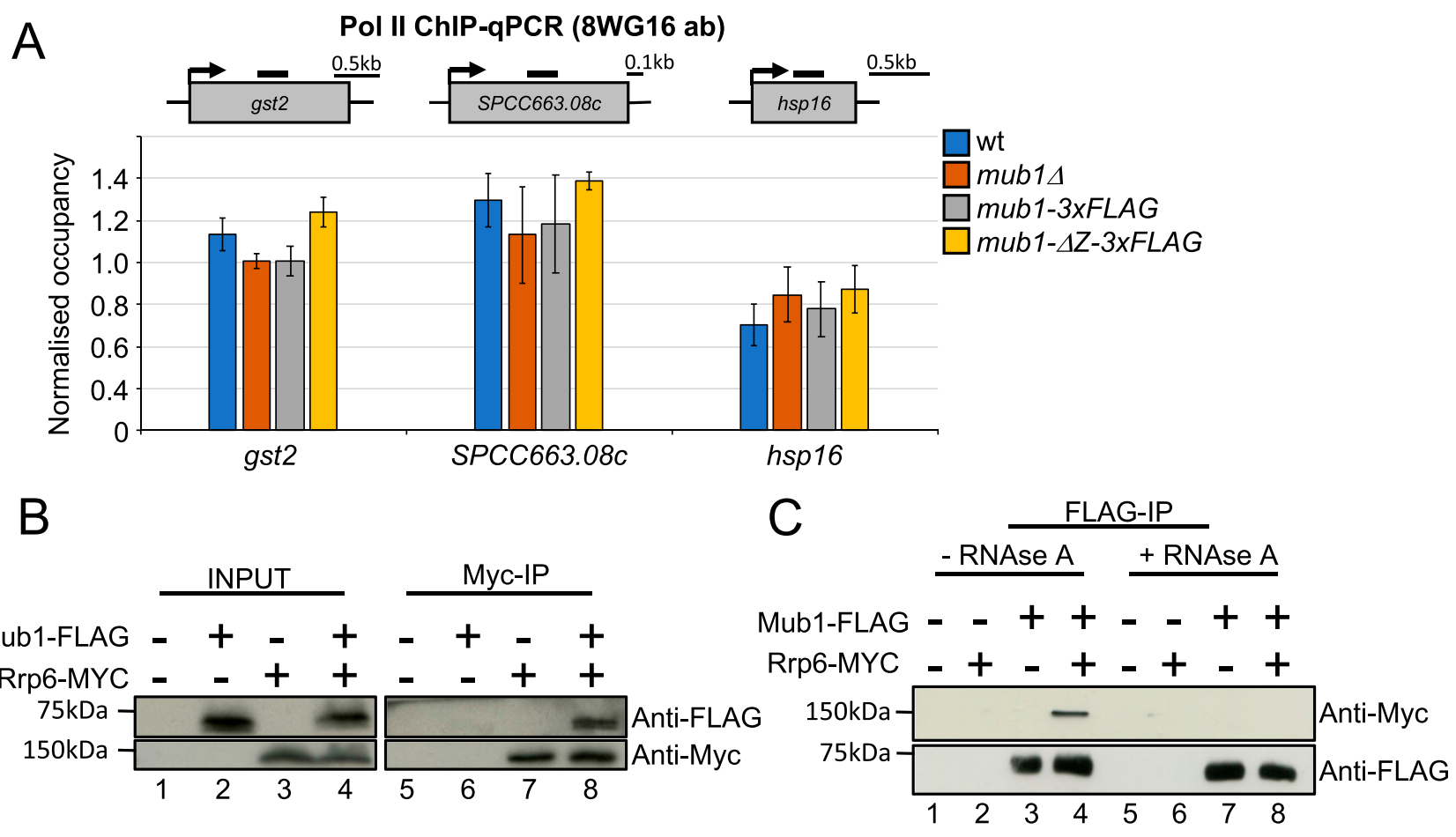

Figure 6. Mub1 regulates stress-dependent genes post-transcriptionally and physically associates with the exosome complex.

(A) ChIP-qPCR analysis of RNA polymerase II (antibody 8WG16) levels at gst2, SPCC663.08c and hsp16 in WT, mub14, mub1-3XFLAG, and mub1- $\triangle$ Z-3XFLAG. The diagram shows the organisation of the genes and the positions of the primer pairs used for qPCR (black bars). ChIP-qPCR quantification shown as the ratio of IP over input relative to a control gene (fbp1). Error bars represent the standard error of the mean (SEM) (biological replicates $n=3$ ). (B) Co-immunoprecipitation of Rrp6-Myc with Mub1-3xFLAG (biological replicates $n=2$ ). $(\mathbf{C})$ Co-immunoprecipitation of Mub1-3xFLAG with Rrp6-Myc from samples that were or were not treated with RNase A, as indicated (biological replicates $n=2$ ).

PCR-generated DNA templates produced from genomic DNA isolated from a wild type S. pombe strain (YP71) with the oligonucleotides listed in Table S11. Probes were added to the membrane and hybridised at $42^{\circ} \mathrm{C}$ overnight. After repeated washes in $2 \times \mathrm{SSC}$, $0.1 \%$ SDS, blots were exposed to Amersham Hyperfilm MP (28-906844; GE Healthcare).

\section{$\operatorname{Poly}(A)+$ RIC}

S. pombe poly(A)+ RIC was performed as described (Kilchert et al, 2020a, 2020b). Briefly, two sets of triplicate experiments (wild-type 1 $(\mathrm{WT1})+m t$ 11-1 = First eXperiment (FX); WT2 + rrp6 $\Delta+$ dis3-54 = Second experiment (SX)) were performed. S. pombe cells were grown at $30^{\circ} \mathrm{C}$ in Edinburgh minimal media supplemented with glutamic acid (EMMG) with limited amounts of uracil (10 $\mathrm{mg} / \mathrm{l})$ and labelled with 4-thiouracil ( $1 \mathrm{mg} / \mathrm{l}$ ) for $4 \mathrm{~h} 30 \mathrm{~min}$. Cells were harvested by filtration, snap-frozen in liquid nitrogen after UV-crosslinking at $3 \mathrm{~J} /$ $\mathrm{cm}^{2}$ in $50 \mathrm{ml}$ PBS and lysed by grinding in liquid nitrogen. The grindate was resuspended in oligo-d(T) lysis buffer (20 mM Tris- $\mathrm{HCl}$, pH 7.5, 500 mM LiCl, 0.5\% Lithium Dodecyl Sulfate [LiDS], 1 mM EDTA; 5 mM DTT, protease inhibitor cocktail IV (fungal) 1:10,000; and 5 mM DTT) and cleared by centrifugation. $1 / 48^{\text {th }}$ of the total volume of WCE was used for proteomics analysis, the rest was subjected to pull-down with oligo-d(T)x25 magnetics beads (NEB-S1419S), $1 \mathrm{ml}$ of slurry per 1 liter of cell culture during $1 \mathrm{~h}$ at $4^{\circ} \mathrm{C}$. Immobilised oligo$d(T) \times 25$ magnetics beads were washed two times with oligo-d(T) wash buffer 1 (20 mM Tris- $\mathrm{HCl}$, pH 7.5, $500 \mathrm{mM}$ LiCl, 0.1\% LiDS, 1 mM EDTA, and $5 \mathrm{mM} \mathrm{DTT}$ ) at $4^{\circ} \mathrm{C}$, two times with oligo-d(T) wash buffer 2 (20 mM Tris- $\mathrm{HCl} \mathrm{pH} \mathrm{7.5,} 500 \mathrm{mM} \mathrm{LiCl}$, and $1 \mathrm{mM}$ EDTA) at room temperature and two times with oligo-d(T) low salt buffer $(20 \mathrm{mM}$ Tris- $\mathrm{HCl}, \mathrm{pH}$ 7.5, $200 \mathrm{mM} \mathrm{LiCl}$, and $1 \mathrm{mM}$ EDTA) at room temperature. RNA-protein complexes were eluted from beads with oligo-d(T) elution buffer ( $20 \mathrm{mM}$ Tris- $\mathrm{HCl}, \mathrm{pH} 7.5$, and $1 \mathrm{mM} \mathrm{EDTA}$ ), $330 \mu \mathrm{l} / \mathrm{l}$ of culture for $10 \mathrm{~min}$ at $55^{\circ} \mathrm{C}$. $1 / 33$ of the oligo-d(T) pull-down total volume was used for RNA sequencing analysis. The rest was subjected to RNase-A and RNase T1 treatment and subjected to mass spectrometry analysis as described in Kilchert et al (2020a).

\section{Statistical data analysis}

Statistical analysis was performed essentially as described (Kilchert et al, 2020a) with the following modifications. To be considered for analysis, proteins were required to be present in at least one of the interactomes with two non-zero values. Raw intensities were $\log _{2}$ transformed, normalised to the same median, and analysis was followed by the imputation of missing values using a minimal value approach (MinDet-where each sample is considered independently). Data manipulations, principal component analysis, and Pearson correlation plots were performed with the DEP package implemented in R (Zhang et al, 2018). Median-normalised data values were used to estimate the log-fold changes between exosome mutants and WT cells, which were further normalised to the 
whole-cell extracts (WCE-normalisation). To minimise batch effects, control experiments (WT cells) were performed twice in triplicates alongside each set of exosome mutants (First triplicate $=$ FX $=$ WT1 + $m$ tl1-1; Second triplicate $=S X=W T 2+r r p 6 \Delta+$ dis3-54). To test the changes between whole-cell extract-normalised (WCE-normalised) proteomes of mutants and WT cells, we used modified scripts from the DEP package. Briefly, this software takes advantage of the Limma package that calculates moderated t-statistics on a linear model fit to the expression data (Zhang et al, 2018). It allows defining custom contrasts (e.g., comparing difference of differences-as in the case of the WCE-normalised intensities). Proteins with a $\log _{2}$ (WCE-normalised RIC of exosome mutant/WCE-normalised RIC of WT) $>1$ were considered to be specifically enriched in exosomes mutants. All other analyses were performed with custom scripts or ones modified from the DEP package. S. pombe GO term annotations and information on individual proteins were retrieved from PomBase (Lock et al, 2019).

\section{Poly(A)+ RNA fluorescence in situ hybridization (FISH)}

Poly(A)+ RNA FISH was carried out as described (Heinrich et al, 2017; Trcek et al, 2017), using an oligo-d(T)x20-alexa488 (7206906; Invitrogen) DNA probe. Briefly, $5 \times 10^{7}-1 \times 10^{8}$ cells were used per hybridization reaction. Cells from an asynchronously growing culture were fixed by the addition of paraformaldehyde to the culture to a final concentration of $4 \%$. The cell pellet was washed with $1 \mathrm{ml}$ of buffer $\mathrm{B}(1.2 \mathrm{M}$ sorbitol and $100 \mathrm{mM} \mathrm{KH} \mathrm{PO}_{4}$ at $\mathrm{pH} 7.5,4^{\circ} \mathrm{C}$ ), immediately resuspended in $1 \mathrm{ml}$ of spheroplast buffer (1.2 M sorbitol, $100 \mathrm{mM} \mathrm{KH}_{2} \mathrm{PO}_{4}$ at pH 7.5, 20 $\mathrm{mM}$ vanadyl ribonuclease complex, and $20 \mu \mathrm{M} \beta$-mercaptoethanol) with 1\% 100T zymolyase (083209-CF; MP Biomedicals) and incubated for $60 \mathrm{~min}$ to digest the cell wall. The reaction was stopped by washing with $1 \mathrm{ml}$ of cold buffer B. Cells were incubated for $20 \mathrm{~min}$ in $0.01 \%$ Triton X-100/1X PBS and washed with $10 \%$ formamide/2× SSC at room temperature. Before hybridization, $50 \mathrm{ng}$ of the oligo- $\mathrm{d}(\mathrm{T})$ probe was mixed with $2 \mu$ l of a 1:1 mixture between yeast transfer RNA $(10 \mathrm{mg} / \mathrm{ml}$, AM7119; Life Technologies) and salmon-sperm DNA (10 mg/ml, 15632011; Life Technologies) and the mixture was dried in a vacuum concentrator. Hybridization buffer $\mathrm{F}\left(20 \%\right.$ formamide, $10 \mathrm{mM} \mathrm{NaHPO}_{4}$ at $\mathrm{pH}$ 7.0; $50 \mu$ l per hybridization) was added, and the probe/ buffer $F$ solution incubated for $3 \mathrm{~min}$ at $95^{\circ} \mathrm{C}$. Buffer $\mathrm{H}(4 \times \mathrm{SSC}, 4 \mathrm{mg} / \mathrm{ml} \mathrm{BSA}$ [acetylated], and $20 \mathrm{mM}$ vanadyl ribonuclease complex; $50 \mu \mathrm{l}$ per hybridization) was added in a 1:1 ratio to the probe/buffer $F$ solution. Cells were resuspended in the mixture and incubated overnight at $37^{\circ} \mathrm{C}$. After three washes ( $10 \%$ formamide, $2 \times$ SSC; $0.1 \%$ Triton X-100, $2 \times$ SSC; and $1 \times$ PBS), cells were resuspended in $1 \times$ PBS/DAPI and mounted on glass slides for imaging. Z-planes spaced by $0.2 \mu \mathrm{m}$ were acquired on an Ultraview spinning-disc confocal with an Olympus UPlanSAPO 100x objective. Acquisition was carried out with DAPI (405 nm) and FITC (488 $\mathrm{nm}$ for alexa488 acquisition) filters.

\section{FISH data analyses}

Images were analysed using Image software (Schneider et al, 2012b). Briefly, Z-stack images (512 × 512 pixels) were generated for DAPI and FISH signal using an average signal intensity stack. Signal intensity was measured across a circle (diameter of 20 pixels) containing the DAPI-stained nucleus or the cytoplasm (DAPInegative, but inside cell). Average intensity of each circle was calculated and the ratio of nuclear signal/cytoplasmic signal calculated for each cell. Mitotic cells (i.e., two DAPI-stained areas inside the same cell) and cells where the circle did not fit in the cytoplasm were excluded from the analysis. The mean and the confidence interval of the mean were calculated with $\alpha=0.05$. Statistical analysis (un-paired $t$ test) was carried out with GraphPad Software. Raw data for each sample and the data used to generate the graph in Figs $3 \mathrm{~B}$ and $4 \mathrm{C}$ are listed in Tables S5 and S6.

\section{RNA sequencing}

For spike-in normalisation, S. cerevisiae cells were added to S. pombe at a 1:10 ratio before RNA isolation. Total RNA was extracted from cultures in mid-log phase using a standard hot phenol method and treated with RNase-free DNase RQ1 (M6101; Promega) to remove DNA. For total RNA sequencing, experiments were performed in duplicates. Ribodepletion was carried out with the ribominus transcriptome isolation kit (K155003; Invitrogen). Poly(A)+ RNA sequencing was performed by using $1 / 33$ of the oligo- $d(T)$ pulldown total volume, subjected to proteinase $\mathrm{K}$ treatment for $1 \mathrm{~h}$ at $50^{\circ} \mathrm{C}$. Poly $(\mathrm{A})+$ RNA was recovered by a standard hot phenol method. Experiments were performed in triplicate. cDNA libraries were prepared using NEBNext Ultra II Directional RNA Library Prep Kit for Illumina (\#E7760S; NEB) for 50 ng of total RNA and using the NEBNext Ultra Directional RNA Library Prep Kit for Illumina (\#E7420; NEB) for 100 ng of WT1, mtl1-1, rrp6 $\Delta$, and dis3-54-purified oligo-d(T) RNA. Paired-end sequencing was carried out on the Illumina HiSeq 500 platform.

\section{RNA-sequencing data analyses}

Quality trimming of sequenced reads was performed using Trimmomatic (Galaxy Version 0.32.3, RRID:SCR_011848). Reads were aligned to the S. pombe genome (ASM294V2.19) using Bowtie 2 (TopHat) (Langmead \& Salzberg, 2012). For spike-in normalisation, reads derived from different S. pombe and S. cerevisiae chromosomes were separated. Reads mapped only once were obtained by SAMTools ( $\mathrm{Li}$ et al, 2009) and reads were mapped to the genome using genome annotation from Eser et al (2016). Differential expression analyses were performed using DESeq2 (Love et al, 2014) in $\mathrm{R}$ and using the spike-in normalisation. For poly(A)+ RNA sequencing, total read count normalisation using DEseq2 (Love et al, 2014) in R was used. The P-values indicated below the Venn diagrams were calculated using a standard Fisher's exact test. For GO analysis, AnGeLi (http:/ / bahlerweb.cs.ucl.ac.uk/ cgi-bin/GLA/GLA_input), a web-based tool, was used (Bitton et al, 2015).

\section{Chromatin immunoprecipitation (ChIP) assay}

Chromatin immunoprecipitation (ChIP) assays were performed as described (Shah et al, 2014). Immunoprecipitations (IPS) were conducted with antibody against Rpb1 (8WG16, 05-952-I; Millipore) coupled to protein G Dynabeads (10004D; Life Technologies). The values correspond to the Rbp1-ChIP signal relative to the input normalised to a control gene ( $f b p 1)$. 


\section{Data Availability}

Raw (fastq) and processed (bedgraph) sequencing data can be downloaded from the National Center for Biotechnology Information (NCBI) Gene Expression Omnibus with the GEO numbers GSE148799 and GSE149187. Mass spectrometry data are available via ProteomeXchange with the identifier PXD016741.

\section{Supplementary Information}

Supplementary Information is available at https://doi.org/10.26508/lsa. 202101111.

\section{Acknowledgements}

We thank the National Bio Resource Yeast Project, S Grewal, P Bernard and JP Javerzat for strains and constructs. We thank members of the Vasilieva laboratory and Jean-Paul Javerzat for their valuable comments on the manuscript. This work was supported by a Wellcome Trust Senior Research fellowship to L Vasiljeva (WT106994/Z/15/Z), and the Emmy Noether Programme of the Deutsche Forschungsgemeinschaft (DFG) to C Kilchert (KI 1657/2-1). We acknowledge the Micron Advanced Bioimaging Unit (supported by the Wellcome Strategic Awards 091911/B/10/Z and 107457/Z/15/Z) for their support and assistance in performing the FISH experiments.

\section{Author Contributions}

A Birot: conceptualization, investigation, visualization, project administration, and writing-original draft, review, and editing. K Kus: formal analysis, investigation, and writing-review and editing.

E Priest: investigation.

A Al Alwash: resources and investigation.

A Castello: methodology and writing-review and editing. S Mohammed: formal analysis, investigation, and methodology.

L Vasiljeva: conceptualization, supervision, funding acquisition, project administration, and writing-original draft, review, and editing.

C Kilchert: conceptualization, investigation, visualization, project administration, and writing-original draft, review, and editing.

\section{Conflict of Interest Statement}

The authors declare that they have no conflict of interest.

\section{References}

Allmang C, Kufel J, Chanfreau G, Mitchell P, Petfalski E, Tollervey D (1999) Functions of the exosome in rRNA, snoRNA and snRNA synthesis. EMBO / 18: 5399-5410. doi:10.1093/emboj/18.19.5399

Andersen PR, Domanski M, Kristiansen MS, Storvall H, Ntini E, Verheggen C, Schein A, Bunkenborg J, Poser I, Hallais M, et al (2013) The human capbinding complex is functionally connected to the nuclear RNA exosome. Nat Struct Mol Biol 20: 1367-1376. doi:10.1038/nsmb.2703
Andrulis ED, Werner J, Nazarian A, Erdjument-Bromage $\mathrm{H}$, Tempst $\mathrm{P}$, Lis JT (2002) The RNA processing exosome is linked to elongating RNA polymerase II in Drosophila. Nature 420: 837-841. doi:10.1038/ nature01181

Atkinson SR, Marguerat S, Bitton DA, Rodríguez-López M, Rallis C, Lemay J-F, Cotobal C, Malecki M, Smialowski P, Mata J, et al (2018) Long noncoding RNA repertoire and targeting by nuclear exosome, cytoplasmic exonuclease, and RNAi in fission yeast. RNA 24: 1195-1213. doi:10.1261/ rna.065524.118

Bähler J, Wu JQ, Longtine MS, Shah NG, McKenzie A, Steever AB, Wach A, Philippsen P, Pringle JR (1998) Heterologous modules for efficient and versatile PCR-based gene targeting in Schizosaccharomyces pombe. Yeast 14: 943-951. doi:10.1002/(SICI)1097-0061(199807)14:10<943::AIDYEA292>3.0.CO;2-Y

Belair C, Sim S, Kim KY, Tanaka Y, Park IH, Wolin SL (2019) The RNA exosome nuclease complex regulates human embryonic stem cell differentiation. J Cell Biol 218: 2564-2582. doi:10.1083/jcb.201811148

Bitton DA, Schubert F, Dey S, Okoniewski M, Smith GC, Khadayate S, Pancaldi V, Wood V, Bähler J (2015) AnGeLi: A tool for the analysis of gene lists from fission yeast. Front Genet 6: 330. doi:10.3389/fgene.2015.00330

Boczonadi V, Müller JS, Pyle A, Munkley J, Dor T, Quartararo J, Ferrero I, Karcagi V, Giunta M, Polvikoski T, et al (2014) EXOSC8 mutations alter mRNA metabolism and cause hypomyelination with spinal muscular atrophy and cerebellar hypoplasia. Nat Commun 5: 4287. doi:10.1038/ ncomms5287

Bühler M, Haas W, Gygi SP, Moazed D (2007) RNAi-dependent and independent RNA turnover mechanisms contribute to heterochromatic gene silencing. Cell 129: 707-721. doi:10.1016/ j.cell.2007.03.038

Bühler M, Spies N, Bartel DP, Moazed D (2008) TRAMP-mediated RNA surveillance prevents spurious entry of RNAs into the Schizosaccharomyces pombe siRNA pathway. Nat Struct Mol Biol 15: 1015-1023. doi:10.1038/nsmb.1481

Castelnuovo M, Zaugg JB, Guffanti E, Maffioletti A, Camblong J, Xu Z, ClauderMünster S, Steinmetz LM, Luscombe NM, Stutz F (2014) Role of histone modifications and early termination in pervasive transcription and antisense-mediated gene silencing in yeast. Nucleic Acids Res 42: 4348-4362. doi:10.1093/nar/gku100

Chen D, Toone WM, Mata J, Lyne R, Burns G, Kivinen K, Brazma A, Jones N, Bähler J (2003) Global transcriptional responses of fission yeast to environmental stress. Mol Biol Cell 14: 214-229. doi:10.1091/mbc.e0208-0499

Chen HM, Futcher B, Leatherwood J (2011) The fission yeast RNA binding protein Mmi1 regulates meiotic genes by controlling intron specific splicing and polyadenylation coupled RNA turnover. PLoS One 6: e26804. doi:10.1371/journal.pone.0026804

Chlebowski A, Lubas M, Jensen TH, Dziembowski A (2013) RNA decay machines: The exosome. Biochim Biophys Acta 1829: 552-560. doi:10.1016/j.bbagrm.2013.01.006

Coy S, Volanakis A, Shah S, Vasiljeva L (2013) The Sm complex is required for the processing of non-coding RNAs by the exosome. PLoS One 8: e65606. doi:10.1371/journal.pone.0065606

Egan ED, Braun CR, Gygi SP, Moazed D (2014) Post-transcriptional regulation of meiotic genes by a nuclear RNA silencing complex. RNA 20: 867-881. doi:10.1261/rna.044479.114

Eser P, Wachutka L, Maier KC, Demel C, Boroni M, lyer S, Cramer P, Gagneur J (2016) Determinants of RNA metabolism in the Schizosaccharomyces pombe genome. Mol Syst Biol 12: 857. doi:10.15252/msb.20156526

Fan J, Kuai B, Wu G, Wu X, Chi B, Wang L, Wang K, Shi Z, Zhang H, Chen S, et al (2017) Exosome cofactor hMTR4 competes with export adaptor ALYREF to ensure balanced nuclear RNA pools for degradation and export. EMBO / 36: 2870-2886. doi:10.15252/embj.201696139 
Fan J, Kuai B, Wang K, Wang L, Wang Y, Wu X, Chi B, Li G, Cheng H (2018) mRNAs are sorted for export or degradation before passing through nuclear speckles. Nucleic Acids Res 46: 8404-8416. doi:10.1093/nar/gky650

Feytout A, Vaur S, Genier S, Vazquez S, Javerzat JP (2011) Psm3 acetylation on conserved lysine residues is dispensable for viability in fission yeast but contributes to Eso1-mediated sister chromatid cohesion by antagonizing Wpl1. Mol Cell Biol 31: 1771-1786. doi:10.1128/ MCB. 01284-10

Garcia-Moreno M, Noerenberg M, Ni S, Järvelin Al, González-Almela E, Lenz CE, Bach-Pages M, Cox V, Avolio R, Davis T, et al (2019) System-wide profiling of RNA-binding proteins uncovers key regulators of virus infection. Mol Cell 74: 196-211.e11. doi:10.1016/j.molcel.2019.01.017

Gaudet P, Livstone MS, Lewis SE, Thomas PD (2011) Phylogenetic-based propagation of functional annotations within the Gene Ontology consortium. Brief Bioinform 12: 449-462. doi:10.1093/bib/bbr042

Gudipati RK, Xu Z, Lebreton A, Séraphin B, Steinmetz LM, Jacquier A, Libri D (2012) Extensive degradation of RNA precursors by the exosome in wild-type cells. Mol Cell 48: 409-421. doi:10.1016/j.molcel.2012.08.018

Harigaya Y, Tanaka H, Yamanaka S, Tanaka K, Watanabe Y, Tsutsumi C, Chikashige Y, Hiraoka Y, Yamashita A, Yamamoto M (2006) Selective elimination of messenger RNA prevents an incidence of untimely meiosis. Nature 442: 45-50. doi:10.1038/nature04881

Hayles J, Wood V, Jeffery L, Hoe KL, Kim DU, Park HO, Salas-Pino S, Heichinger C, Nurse P (2013) A genome-wide resource of cell cycle and cell shape genes of fission yeast. Open Biol 3: 130053. doi:10.1098/rsob.130053

Heinrich S, Sidler CL, Azzalin CM, Weis K (2017) Stem-loop RNA labeling can affect nuclear and cytoplasmic mRNA processing. RNA 23: 134-141. doi:10.1261/rna.057786.116

Hirose M, Tohda H, Giga-Hama Y, Tsushima R, Zako T, lizuka R, Pack C, Kinjo M, Ishii N, Yohda M (2005) Interaction of a small heat shock protein of the fission yeast, Schizosaccharomyces pombe, with a denatured protein at elevated temperature. J Biol Chem 280: 32586-32593. doi:10.1074/ jbc.M504121200

Houseley J, Tollervey D (2009) The many pathways of RNA degradation. Cell 136: 763-776. doi:10.1016/j.cell.2009.01.019

Isken O, Maquat LE (2007) Quality control of eukaryotic mRNA: Safeguarding cells from abnormal mRNA function. Genes Dev 21: 1833-1856. doi:10.1101/gad.1566807

Januszyk K, Lima CD (2014) The eukaryotic RNA exosome. Curr Opin Struct Biol 24: 132-140. doi:10.1016/j.sbi.2014.01.011

Keller C, Woolcock K, Hess D, Bühler M (2010) Proteomic and functional analysis of the noncanonical poly(A) polymerase Cid14. RNA 16: 1124-1129. doi:10.1261/rna.2053710

Kilchert C, Wittmann S, Passoni M, Shah S, Granneman S, Vasiljeva L (2015) Regulation of mRNA levels by decay-promoting introns that recruit the exosome specificity factor Mmi1. Cell Rep 13: 2504-2515. doi:10.1016/j.celrep.2015.11.026

Kilchert C, Wittmann S, Vasiljeva L (2016) The regulation and functions of the nuclear RNA exosome complex. Nat Rev Mol Cell Biol 17: 227-239. doi:10.1038/nrm.2015.15

Kilchert C, Kecman T, Priest E, Hester S, Aydin E, Kus K, Rossbach O, Castello A, Mohammed S, Vasiljeva L (2020a) System-wide analyses of the fission yeast poly(A)+ RNA interactome reveal insights into organization and function of RNA-protein complexes. Genome Res 30: 1012-1026. doi:10.1101/gr.257006.119

Kilchert C, Hester S, Castello A, Mohammed S, Vasiljeva L (2020b) Comparative poly(A)+ RNA interactome capture of RNA surveillance mutants. Methods Mol Biol 2062: 255-276. doi:10.1007/978-1-4939-9822-7_13

Kiss DL, Andrulis ED (2010) Genome-wide analysis reveals distinct substrate specificities of Rrp6, Dis3, and core exosome subunits. RNA 16: 781-791. doi:10.1261/rna.1906710
LaCava J, Houseley J, Saveanu C, Petfalski E, Thompson E, Jacquier A, Tollervey D (2005) RNA degradation by the exosome is promoted by a nuclear polyadenylation complex. Cell 121: 713-724. doi:10.1016/j.cell. 2005.04.029

Langmead B, Salzberg SL (2012) Fast gapped-read alignment with Bowtie 2. Nat Methods 9: 357-359. doi:10.1038/nmeth.1923

Lee NN, Chalamcharla VR, Reyes-Turcu F, Mehta S, Zofall M, Balachandran V, Dhakshnamoorthy J, Taneja N, Yamanaka S, Zhou M, et al (2013) Mtr4like protein coordinates nuclear RNA processing for heterochromatin assembly and for telomere maintenance. Cell 155: 1061-1074. doi:10.1016/j.cell.2013.10.027

Lemay JF, Larochelle M, Marguerat S, Atkinson S, Bähler J, Bachand F (2014) The RNA exosome promotes transcription termination of backtracked RNA polymerase II. Nat Struct Mol Biol 21: 919-926. doi:10.1038/ nsmb.2893

Li H, Handsaker B, Wysoker A, Fennell T, Ruan J, Homer N, Marth G, Abecasis G, Durbin R (2009) The sequence alignment/map format and SAMtools. Bioinformatics 25: 2078-2079. doi:10.1093/bioinformatics/btp352

Lingaraju M, Johnsen D, Schlundt A, Langer LM, Basquin J, Sattler M, Heick Jensen T, Falk S, Conti E (2019) The MTR4 helicase recruits nuclear adaptors of the human RNA exosome using distinct arch-interacting motifs. Nat Commun 10: 3393. doi:10.1038/s41467-019-11339-x

Liu J, Dou X, Chen C, Chen C, Liu C, Xu MM, Zhao S, Shen B, Gao Y, Han D, et al (2020) N 6-methyladenosine of chromosome-associated regulatory RNA regulates chromatin state and transcription. Science 367: 580-586. doi:10.1126/science.aay6018

Lloret-Llinares M, Karadoulama E, Chen Y, Wojenski LA, Villafano GJ, Bornholdt J, Andersson R, Core L, Sandelin A, Jensen TH (2018) The RNA exosome contributes to gene expression regulation during stem cell differentiation. Nucleic Acids Res 46: 11502-11513. doi:10.1093/nar/ gky817

Lock A, Rutherford K, Harris MA, Hayles J, Oliver SG, Bähler J, Wood V (2019) PomBase 2018: User-driven reimplementation of the fission yeast database provides rapid and intuitive access to diverse, interconnected information. Nucleic Acids Res 47: D821-D827. doi:10.1093/nar/gky961

Love MI, Huber W, Anders S (2014) Moderated estimation of fold change and dispersion for RNA-seq data with DESeq2. Genome Biol 15: 550. doi:10.1186/s13059-014-0550-8

Lunde BM, Moore C, Varani G (2007) RNA-binding proteins: Modular design for efficient function. Nat Rev Mol Cell Biol 8: 479-490. doi:10.1038/ nrm2178

Makino DL, Baumgärtner M, Conti E (2013) Crystal structure of an RNA-bound 11-subunit eukaryotic exosome complex. Nature 495: 70-75. doi:10.1038/nature11870

Mclver SC, Kang YA, DeVilbiss AW, O'Driscoll CA, Ouellette JN, Pope NJ, Camprecios G, Chang C), Yang D, Bouhassira EE, et al (2014) The exosome complex establishes a barricade to erythroid maturation. Blood 124: 2285-2297. doi:10.1182/blood-2014-04-571083

Mclver SC, Katsumura KR, Davids E, Liu P, Kang Y-A, Yang D, Bresnick EH (2016) Exosome complex orchestrates developmental signaling to balance proliferation and differentiation during erythropoiesis. Elife 5: e17877. doi:10.7554/eLife.17877

Meola N, Domanski M, Karadoulama E, Chen Y, Gentil C, Pultz D, VittingSeerup K, Lykke-Andersen S, Andersen JS, Sandelin A, et al (2016) Identification of a nuclear exosome decay pathway for processed transcripts. Mol Cell 64: 520-533. doi:10.1016/j.molcel.2016.09.025

Mitchell P, Petfalski E, Shevchenko A, Mann M, Tollervey D (1997) The exosome: A conserved eukaryotic RNA processing complex containing multiple $3^{\prime} \rightarrow 5^{\prime}$ exoribonucleases. Cell 91: 457-466. doi:10.1016/s0092$8674(00) 80432-8$ 
Moreno S, Klar A, Nurse P (1991) Molecular genetic analysis of fission yeast Schizosaccharomyces pombe. Methods Enzymol 194: 795-823. doi:10.1016/0076-6879(91)94059-l

Murakami H, Goto DB, Toda T, Chen ES, Grewal SI, Martienssen RA, Yanagida M (2007) Ribonuclease activity of Dis3 is required for mitotic progression and provides a possible link between heterochromatin and kinetochore function. PLoS One 2: e317. doi:10.1371/ journal.pone.0000317

Mure F, Corbin A, Benbahouche NEH, Bertrand E, Manet E, Gruffat H (2018) The splicing factor SRSF3 is functionally connected to the nuclear RNA exosome for intronless mRNA decay. Sci Rep 8: 12901. doi:10.1038/ s41598-018-31078-1

Nag A, Steitz JA (2012) Tri-snRNP-associated proteins interact with subunits of the TRAMP and nuclear exosome complexes, linking RNA decay and pre-mRNA splicing. RNA Biol 9: 334-342. doi:10.4161/rna.19431

Neil H, Malabat C, d'Aubenton-Carafa Y, Xu Z, Steinmetz LM, Jacquier A (2009) Widespread bidirectional promoters are the major source of cryptic transcripts in yeast. Nature 457: 1038-1042. doi:10.1038/nature07747

Ohkura H, Adachi Y, Kinoshita N, Niwa O, Toda T, Yanagida M (1988) Coldsensitive and caffeine-supersensitive mutants of the Schizosaccharomyces pombe dis genes implicated in sister chromatid separation during mitosis. EMBO J 7: 1465-1473. doi:10.1002/ j.1460-2075.1988.tb02964.x

Paul B, Montpetit B (2016) Altered RNA processing and export lead to retention of mRNAs near transcription sites and nuclear pore complexes or within the nucleolus. Mol Biol Cell 27: 2742-2756. doi:10.1091/mbc.E16-04-0244

Preker P, Nielsen J, Kammler S, Lykke-Andersen S, Christensen MS, Mapendano CK, Schierup MH, Jensen TH (2008) RNA exosome depletion reveals transcription upstream of active human promoters. Science 322: 1851-1854. doi:10.1126/science.1164096

Schneider C, Kudla G, Wlotzka W, Tuck A, Tollervey D (2012a) Transcriptomewide analysis of exosome targets. Mol Cell 48: 422-433. doi:10.1016/ j.molcel.2012.08.013

Schneider CA, Rasband WS, Eliceiri KW (2012b) NIH image to Image): 25 years of image analysis. Nat Methods 9: 671-675. doi:10.1038/nmeth.2089

Shah S, Wittmann S, Kilchert C, Vasiljeva L (2014) IncRNA recruits RNAi and the exosome to dynamically regulate pho1 expression in response to phosphate levels in fission yeast. Genes Dev 28: 231-244. doi:10.1101/ gad.230177.113

Shichino Y, Otsubo Y, Yamamoto M, Yamashita A (2020) Meiotic gene silencing complex MTREC/NURS recruits the nuclear exosome to YTHRNA-binding protein Mmi1. PLoS Genet 16: e1008598. doi:10.1371/ journal.pgen.1008598

Shiomi T, Fukushima K, Suzuki N, Nakashima N, Noguchi E, Nishimoto T (1998) Human dis3p, which binds to either GTP- or GDP-Ran, complements Saccharomyces cerevisiae dis3. J Biochem 123: 883-890. doi:10.1093/ oxfordjournals.jbchem.a022020

Silla T, Karadoulama E, Mąkosa D, Lubas M, Jensen TH (2018) The RNA exosome adaptor $\mathrm{ZFC} 3 \mathrm{H} 1$ functionally competes with nuclear export activity to retain target transcripts. Cell Rep 23: 2199-2210. doi:10.1016/ j.celrep.2018.04.061

St-André O, Lemieux C, Perreault A, Lackner DH, Bähler J, Bachand F (2010) Negative regulation of meiotic gene expression by the nuclear poly(a)-binding protein in fission yeast. J Biol Chem 285: 27859-27868. doi:10.1074/jbc.M110.150748

Sugiyama T, Sugioka-Sugiyama R (2011) Red1 promotes the elimination of meiosis-specific mRNAs in vegetatively growing fission yeast. EMBO J 30: 1027-1039. doi:10.1038/emboj.2011.32

Sugiyama T, Wanatabe N, Kitahata E, Tani T, Sugioka-Sugiyama R (2013) Red5 and three nuclear pore components are essential for efficient suppression of specific mRNAs during vegetative growth of fission yeast. Nucleic Acids Res 41: 6674-6686. doi:10.1093/nar/gkt363
Szczepińska T, Kalisiak K, Tomecki R, Labno A, Borowski LS, Kulinski TM, Adamska D, Kosinska J, Dziembowski A (2015) DIS3 shapes the RNA polymerase II transcriptome in humans by degrading a variety of unwanted transcripts. Genome Res 25: 1622-1633. doi:10.1101/ gr.189597.115

Tatomer DC, Elrod ND, Liang D, Xiao MS, Jiang JZ, Jonathan M, Huang KL, Wagner EJ, Cherry S, Wilusz JE (2019) The Integrator complex cleaves nascent mRNAs to attenuate transcription. Genes Dev 33: 1525-1538. doi:10.1101/gad.330167.119

Telekawa C, Boisvert FM, Bachand F (2018) Proteomic profiling and functional characterization of post-translational modifications of the fission yeast RNA exosome. Nucleic Acids Res 46: 11169-11183. doi:10.1093/ nar/gky915

Thiebaut M, Kisseleva-Romanova E, Rougemaille M, Boulay J, Libri D (2006) Transcription termination and nuclear degradation of cryptic unstable transcripts: A role for the nrd1-nab3 pathway in genome surveillance. Mol Cell 23: 853-864. doi:10.1016/j.molcel.2006.07.029

Tomecki R, Dziembowski A (2010) Novel endoribonucleases as central players in various pathways of eukaryotic RNA metabolism. RNA 16: 1692-1724. doi:10.1261/rna.2237610

Tomecki R, Kristiansen MS, Lykke-Andersen S, Chlebowski A, Larsen KM, Szczesny RJ, Drazkowska K, Pastula A, Andersen JS, Stepien PP, et al (2010) The human core exosome interacts with differentially localized processive RNases: hDIS3 and hDIS3L. EMBO / 29: 2342-2357. doi:10.1038/emboj.2010.121

Trcek T, Lionnet T, Shroff H, Lehmann R (2017) mRNA quantification using single-molecule FISH in Drosophila embryos. Nat Protoc 12: 1326-1348. doi:10.1038/nprot.2017.030

Tseng C-K, Wang H-F, Schroeder MR, Baumann P (2018) The H/ACA complex disrupts triplex in hTR precursor to permit processing by RRP6 and PARN. Nat Commun 9: 5430. doi:10.1038/s41467-018-07822-6

Tudek A, Porrua O, Kabzinski T, Lidschreiber M, Kubicek K, Fortova A, Lacroute F, Vanacova S, Cramer P, Stefl R, et al (2014) Molecular basis for coordinating transcription termination with noncoding RNA degradation. Mol Cell 55: 467-481. doi:10.1016/j.molcel.2014.05.031

Vanácová S, Wolf J, Martin G, Blank D, Dettwiler S, Friedlein A, Langen H, Keith G, Keller W (2005) A new yeast poly(A) polymerase complex involved in RNA quality control. PLOS Biol 3: e189. doi:10.1371/journal. pbio.0030189

Vasiljeva L, Buratowski S (2006) Nrd1 interacts with the nuclear exosome for 3' processing of RNA polymerase II transcripts. Mol Cell 21: 239-248. doi:10.1016/j.molcel.2005.11.028

Vasiljeva L, Kim M, Terzi N, Soares LM, Buratowski S (2008a) Transcription termination and RNA degradation contribute to silencing of RNA polymerase II transcription within heterochromatin. Mol Cell 29: 313-323. doi:10.1016/j.molcel.2008.01.011

Vasiljeva L, Kim M, Mutschler H, Buratowski S, Meinhart A (2008b) The Nrd1Nab3-Sen1 termination complex interacts with the Ser5phosphorylated RNA polymerase II C-terminal domain. Nat Struct Mol Biol 15: 795-804. doi:10.1038/nsmb.1468

Vo TV, Dhakshnamoorthy J, Larkin M, Zofall M, Thillainadesan G, Balachandran V, Holla S, Wheeler D, Grewal SIS (2019) CPF recruitment to non-canonical transcription termination sites triggers heterochromatin assembly and gene silencing. Cell Rep 28 : 267-281.e5. doi:10.1016/j.celrep.2019.05.107

Wan J, Yourshaw M, Mamsa H, Rudnik-Schöneborn S, Menezes MP, Hong JE, Leong DW, Senderek J, Salman MS, Chitayat D, et al (2012) Mutations in the RNA exosome component gene EXOSC3 cause pontocerebellar hypoplasia and spinal motor neuron degeneration. Nat Genet 44: 704-708. doi:10.1038/ng.2254

Wang C, Liu Y, DeMario SM, Mandric I, Gonzalez-Figueroa C, Chanfreau GF (2020) Rrp6 moonlights in an RNA exosome-independent manner to 
promote cell survival and gene expression during stress. Cell Rep 31: 107754. doi:10.1016/j.celrep.2020.107754

Weick EM, Lima CD (2020) RNA helicases are hubs that orchestrate exosomedependent 3'-5' decay. Curr Opin Struct Biol 67: 86-94. doi:10.1016/ j.sbi.2020.09.010

Weick EM, Puno MR, Januszyk K, Zinder JC, DiMattia MA, Lima CD (2018) Helicase-dependent RNA decay illuminated by a cryo-EM structure of a human nuclear RNA exosome-MTR4 complex. Cell 173: 1663-1677.e21. doi:10.1016/j.cell.2018.05.041

Wyers F, Rougemaille M, Badis G, Rousselle JC, Dufour ME, Boulay J, Régnault B, Devaux F, Namane A, Séraphin B, et al (2005) Cryptic pol II transcripts are degraded by a nuclear quality control pathway involving a new poly(A) polymerase. Cell 121: 725-737. doi:10.1016/ j.cell.2005.04.030

Yamashita A, Shichino Y, Tanaka H, Hiriart E, Touat-Todeschini L, Vavasseur A, Ding DQ, Hiraoka Y, Verdel A, Yamamoto M (2012) Hexanucleotide motifs mediate recruitment of the RNA elimination machinery to silent meiotic genes. Open Biol 2: 120014. doi:10.1098/rsob.120014

Yamashita A, Takayama T, Iwata R, Yamamoto M (2013) A novel factor Iss10 regulates Mmi1-mediated selective elimination of meiotic transcripts. Nucleic Acids Res 41: 9680-9687. doi:10.1093/nar/ gkt763

Yang X, Bayat V, DiDonato N, Zhao Y, Zarnegar B, Siprashvili Z, Lopez-Pajares V, Sun T, Tao S, Li C, et al (2020) Genetic and genomic studies of pathogenic EXOSC2 mutations in the newly described disease SHRF implicate the autophagy pathway in disease pathogenesis. Hum Mol Genet 29: 541-553. doi:10.1093/hmg/ddz251

Zhang X, Smits AH, van Tilburg GB, Ovaa H, Huber W, Vermeulen M (2018) Proteome-wide identification of ubiquitin interactions using UbIAMS. Nat Protoc 13: 530-550. doi:10.1038/nprot.2017.147

Zhou Y, Zhu J, Schermann G, Ohle C, Bendrin K, Sugioka-Sugiyama R, Sugiyama T, Fischer T (2015) The fission yeast MTREC complex targets CUTs and unspliced pre-mRNAs to the nuclear exosome. Nat Commun 6: 7050-7111. doi:10.1038/ncomms8050

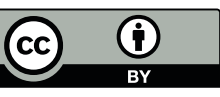

License: This article is available under a Creative Commons License (Attribution 4.0 International, as described at https://creativecommons.org/ licenses/by/4.0/). 